\title{
Hydrodynamic forces in water exit problems
}

\author{
A.A. Korobkin ${ }^{a}$, T.I. Khabakhpasheva ${ }^{a 1}, K_{\text {Kevin J. Maki }}{ }^{b}$ \\ a School of Mathematics, University of East Anglia, Norwich, NR4 7TJ, UK \\ $b$ Department of Naval Architecture and Marine Engineering University of Michigan
}

Ann Arbor, MI, 48109 USA

\begin{abstract}
The three-dimensional steady problem of an elongated smooth body moving along the water free surface at a constant speed is considered within the $2 \mathrm{D}+\mathrm{T}$ approximation. The corresponding unsteady two-dimensional problem describes both the water entry and the subsequent exit of a smooth contour from the water. The shape of the contour varies in time. The present paper is concerned with the exit stage. The draft of the body is small compared with the body length and beam. The hydrodynamic loads during the entry stage are evaluated by the original Wagner model of water impact. The linearized exit model [5] is generalised to account for time-dependent acceleration of the body and the body shape which also varies in time. The integral equation with respect to the size of the wetted area of the body is solved numerically. The theoretical predictions of the hydrodynamic forces acting on the body during its exit from the liquid are compared with the numerical results obtained by solving the Navier-Stokes equations. A simplified model of water exit with the body shape approximated by a parabolic contour with a time-dependent radius of curvature is proposed and validated. It is shown that the linearized water-exit model with non-linear correction terms predicts reasonably well the hydrodynamic loads.
\end{abstract}

Key words: Water exit, Fluid-structure interaction, Hydrodynamic force, CFD

\section{Introduction}

The present study is motivated by hydrodynamics of high-speed vessels and aircraft ditching on the water surface, where the wetted part of the hull is streamlined and the hull is elongated in the direction of the motion. Hydrodynamic loads over the wetted part of the elongated hull are estimated by using the $2 \mathrm{D}+\mathrm{T}$ approximation [6]. In this approximation, the three-dimensional nonlinear stationary problem is reduced to a two-dimensional unsteady problem of water entry and exit. The latter problem can be linearized if the draft of the body and the local deadrise angle of the body cross section are both small. In particular, if the draft of the body is much smaller that the beam of the body. For the stationary three-dimensional problem of a smooth body moving at a constant speed along the water surface, we introduce a vertical plane perpendicular to the direction of the body motion and consider the unsteady two-dimensional flow within this plane caused by the body passing through the plane. The intersection of the moving body surface with this control plane provides a two-dimensional contour which changes its shape in time and penetrates the water surface. For a three-dimensional body with smooth surface the entry stage ends when the two-dimensional contour stops expanding. During the next stage, which is referred to below as the exit stage, the contour contracts and exits from the water. The entry stage was investigated in [8] by the modified Logvinovich model [9].

\footnotetext{
${ }^{1}$ Corresponding author.

E-mail address: T.Khabakhpasheva@uea.ac.uk (T.I. Khabakhpasheva)
} 
It was found that the theoretical results are very close to the computational fluid dynamics (CFD) results obtained by numerical simulations of the Navier-Stokes equations (see [10]-[12] for details of the simulations). During the exit stage, the von Karman model was used in [8]. However, the theoretical results from [8] for the exit stage were not as accurate as for the entry stage. Recently a linearized exit model was developed in [5]. Only symmetric rigid contours moving from the water surface with a constant acceleration were considered. The exit model was formulated in terms of the linearized pressure with the additional condition that the speed of the contact points is proportional to the local speed of the flow. This model does not account for the shape of the body but still corresponds quite well to the CFD results from [10]. The model was developed further in [14] to account for a variable in time acceleration of the body. The bodies in [5] and [14] were rigid, symmetric and only vertical motions were allowed. The two-dimensional problem of a body whose shape varies in time was studied in [8] for an expanding and contracting circular cylinder. The numerical and theoretical forces were very close to each other during the expansion (entry) stage but rather different during the contraction (exit) stage.

The forced constant acceleration exit of two-dimensional bodies through a free-surface was numerically investigated in [15]. Cases of symmetric wedges, asymmetric wedges, truncated wedges and boxes were considered. The problem was solved numerically by means of a boundary-element method. The analytical added-mass force acting on a body just after the body started to move was presented for the exit of symmetric wedges and boxes with constant acceleration using conformal mappings. The numerical and analytical results for added-mass force were compared and give good agreement for the exit of a symmetric wedge but only moderate agreement for the box.

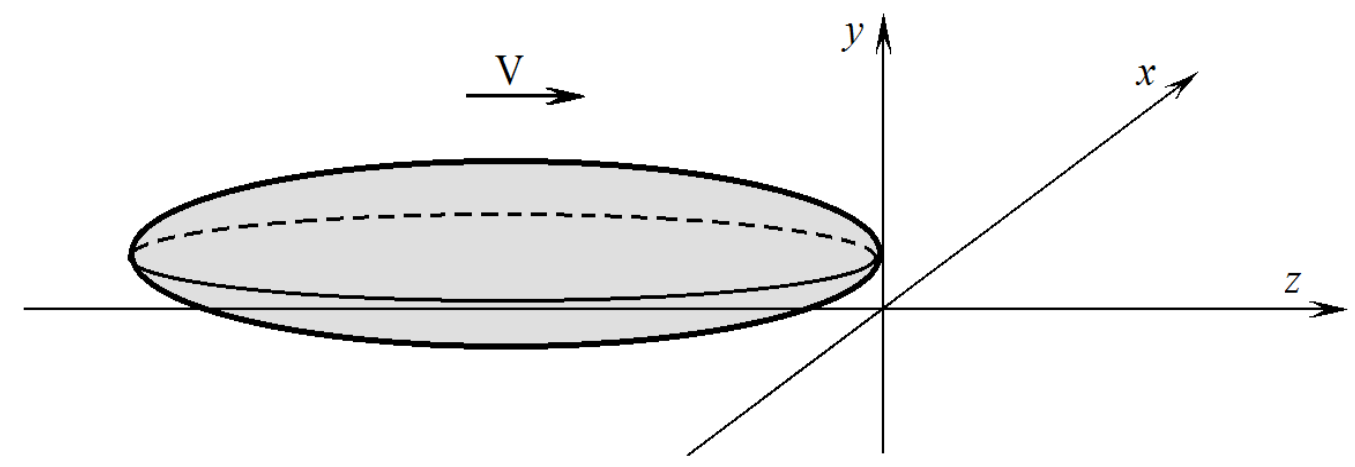

Fig. 1 Sketch of an elongated body moving along the water free surface at a fixed penetration depth.

There have been numerous recent authors to study impact problems using fully nonlinear CFD-based techniques. The OpenFOAM opensource CFD toolkit is used in $[2,3]$ to study the impact of rigid bodies with the calm-water surface. Results are provided for pressure, force, and energy of the body during impact, and the numerical predictions are validated with experimental measurements. In [1] CFD and the commercial structuralanalysis software ABAQUS are employed to study the impact of an elastic plate with the water surface. A review of the capabilities of CFD to predict the impact of a wedgeshaped body into the water surface can be found in [4]. All of these references focus on impact, whereas the present work addresses specifically the exit phase of bodies from the water. 
In the present paper, we apply the exit model from [5] to the bodies of varying shapes and introduce some correction terms to the hydrodynamic loads to account for the shape of the body (see [8]) and nonlinear effects. The entry stage is considered below within the original Wagner theory of water impact. It is known (see [8]) that the modified Logvinovich model [9] provides better predictions of the hydrodynamic loads during the entry but here we are mainly concerned with the negative loads during the exit stage.

The linearized two-dimensional problem of water entry and exit is formulated in Section 2. The relation of this problem to the three-dimensional problem of a smooth body moving along water surface is explained. The CFD method is presented in Section 3. The hydrodynamic force acting on a two-dimensional body, which enters the water with a time-dependent speed and whose shape varies in time, is presented in Section 4. The linearized model of water exit from [5] is reviewed in Section 5 together with the conditions of its applicability and generalised to the case of non-constant acceleration of the body. The water-exit model is generalised to the problem of varying in time shape of the body in Section 6. The theoretical predictions are compared with the numerical solutions of the Navier-Stokes equations and with the solutions of the exit problems for parabolic contours. To improve the theoretical predictions, the shape of the body and some non-linear terms in the Bernoulli equation for the hydrodynamic pressure are taken into account within a weakly nonlinear model of water exit in Section 7 . The obtained results and developed models are applied in Section 8 to the steady problem of the ellipsoid moving along the water surface with a small draft. The findings of the analysis are summarized and conclusions are drawn in Section 9.

\section{Formulation of water entry and exit problems}

The three-dimensional problem of a smooth body moving horizontally along the water free surface at the fixed penetration depth is considered in the Cartesian coordinate system $x, y, z$, where the $z$-axis is in the direction of the body motion and the plane $y=0$ corresponds to the equilibrium water level (Figure 1). The origin of the coordinate system is placed on the equilibrium free surface. The nose of the body touches the plane $z=0$ at a time instant taken as the initial one, $t=0$. The elevation of the free surface in front of the moving body is assumed to be zero. This is a drawback of the $2 \mathrm{D}+\mathrm{T}$ model used in this study. More details about the local flow in the region of the bow can be found in $[6,7]$.

The liquid is assumed to be inviscid, incompressible and of infinite depth. The flow caused by the moving body is potential, steady and three-dimensional. The body surface is smooth. The body is elongated in the direction of its motion. The speed of the body is constant. Gravity and surface tension effects are not taken into account in calculations of the hydrodynamic loads acting on the body, which is a right approximation for large dimensions of a body moving at a relatively high speed. The plane $z=0$ is treated here as a control plane (see [8]). Note that the position of the control plane is arbitrary for the body moving with a constant speed. The flow within this plane is assumed twodimensional, which is an acceptable approximation for a three-dimensional body elongated

in the direction of its motion. The suitability of this assumption depends on how the vertical sections of the body vary along the length, which is slowly for elongated bodies, with exceptions limited to regions near the very front and back (see [6] and [8] for more details). Within the control plane, $z=0$, the $2 \mathrm{D}$ flow is caused by the moving contour 
which corresponds to the intersection curve between the surface of the moving body and the plane $z=0$. The contour is described by the equation

$$
y=y_{b}(x, t),
$$

where the function $y_{b}(x, t)$ is known. The function $y_{b}(x, t)$ is assumed smooth and even, $y_{b}(-x, t)=y_{b}(x, t), y_{b}(0, t)=0$. For a body of length $L_{b}$ moving with speed $V$, the time interval of the body-fluid interaction is $0<t<L_{b} / V$ within the $2 \mathrm{D}+\mathrm{T}$ approximation. This time interval is subdivided into four stages (see Figure 2): (1) $0<t<t_{0}$, the contour (1) is still above the water surface and there is no flow in the control plane; (2) $t_{0}<t<t_{1}$, during this stage the contour penetrates the water and its wetted area expands, this stage is referred to as the entry stage; (3) $t_{1}<t<t_{2}$, during this stage the wetted area shrinks in time and the body surface is completely dry at $t=t_{2}$, this stage is referred to as the exit stage; (4) $t>t_{2}$, after the end of the exit stage the flow in the control plane continues with the body being above the water surface, this flow is not taken into account in calculations of the hydrodynamic loads acting on the three-dimensional body within the $2 \mathrm{D}+\mathrm{T}$ approximation.
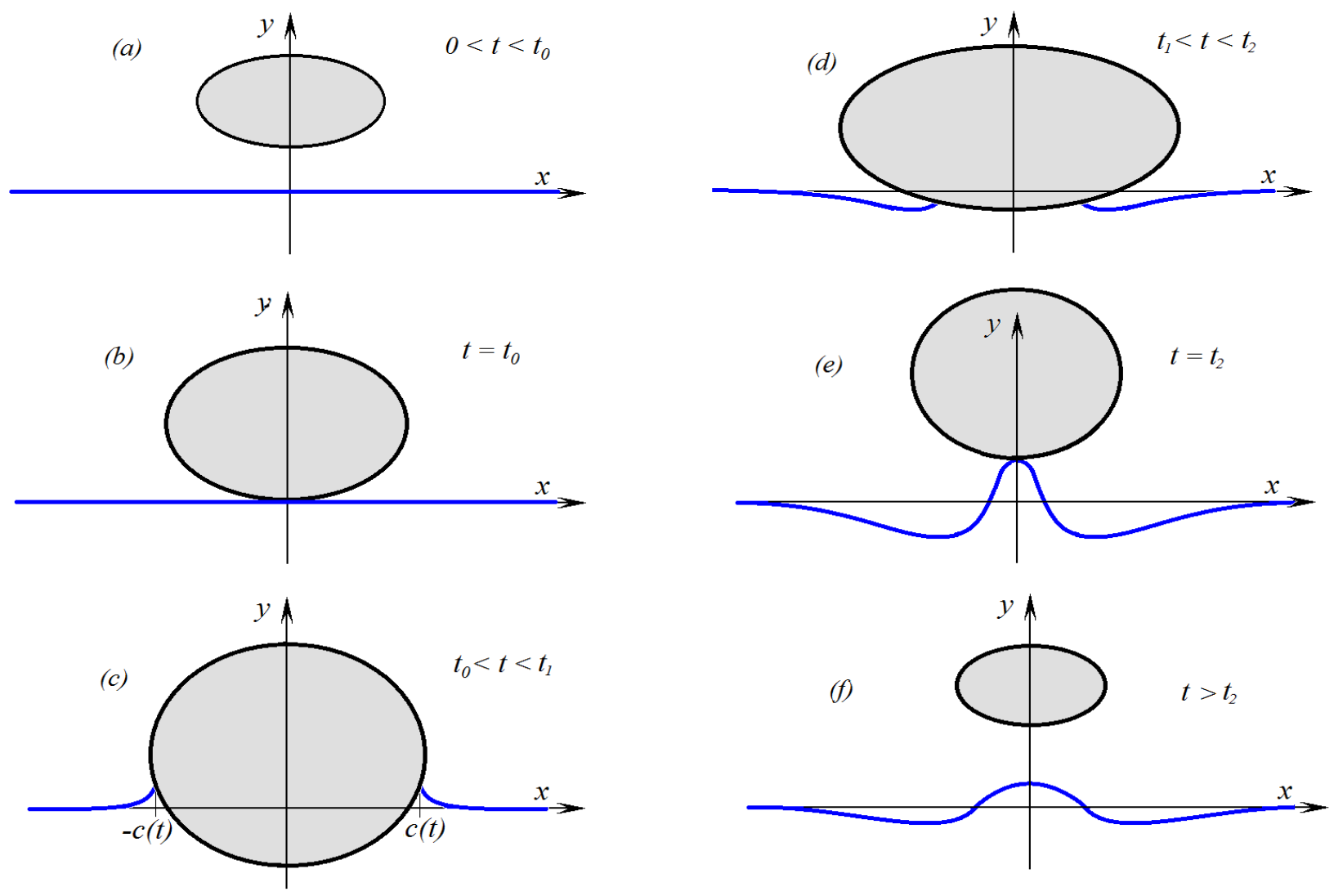

Fig. 2 Four stages of the flow in the control plane:

(a) The body appears in the control plane but is above the water surface;

(b) The $2 \mathrm{D}$ body touches the undisturbed free surface at a single point at $t=t_{0}$;

(c) the body penetrates the water during the entry stage, $t_{0}<t<t_{1}$;

(d) the wetted part of the body surface shrinks during the exit stage, $t_{1}<t<t_{2}$;

(e) the body surface touches the elevated water at a single point at the end of the exit stage;

(f) the body is above the water surface and continues shrinking after $t_{2}$.

The problem of the flow in the control plane is considered under the assumption that the linear size of the body cross sections, $L_{c}$, is much greater than the penetration depth 
of the body, $h_{0}$, but much smaller than the length of the body, $L_{b}$, introduced above. The characteristic time $T$ of both the entry and exit stages is estimated as $L_{b} / V$ and the velocity of the contour in the control plane is estimated as $h_{0} / T=h_{0} V / L_{b}$. The scale of the velocity potential $\varphi(x, y, t)$ is $L_{s} h_{0} / T$ and the linear scale is $L_{c}$. The hydrodynamic pressure $p$ in the control plane, which is used to evaluate the sectional loads acting on the moving body, is given by the Bernoulli equation

$$
p(x, y, t)=-\rho\left(\varphi_{t}+\frac{1}{2}|\nabla \varphi|^{2}\right),
$$

where $|\nabla \varphi|^{2} / \varphi_{t}=O\left(\left(h_{0} / T\right)^{2} /\left(L_{s} h_{0} / T^{2}\right)\right)=O\left(h_{0} / L_{s}\right)$ and $\rho$ is the liquid density. Therefore, the nonlinear term in the Bernoulli equation (2) can be neglected if the penetration depth is small, $h_{0} / L_{c} \ll 1$. Correspondingly, the elevation of the free surface $\eta(x, t)$ caused by the moving body is scaled with $h_{0}$ and can be neglected compared with the characteristic length, $L_{c}$, of the flow in the control plane. As a result, the boundary conditions on both the liquid free surface and on the wetted part of the contour, $y=y_{b}(x, t)$, can be linearized and imposed on the equilibrium position of the water surface.

The linear boundary-value problem with respect to the velocity potential $\varphi(x, y, t)$ is given by

$$
\begin{array}{cc}
\nabla^{2} \varphi=0 & (y<0), \\
\varphi_{t}=0 & (y=0,|x|>c(t)), \\
\varphi_{y}=y_{b, t}(x, t) & (y=0,|x|<c(t)), \\
\varphi \rightarrow 0 & \left(x^{2}+y^{2} \rightarrow \infty\right),
\end{array}
$$

where the positions of the contact points, $x= \pm c(t)$, in the symmetric case, are described by a single function $c(t)$. This function is unknown in advance and should be determined as part of the solution. Initially, $c\left(t_{0}\right)=0$. During the entry stage, $t_{0}<t<t_{1}$, the contact region, $|x|<c(t)$, between the liquid and the section of the body expands with $d c / d t$ being positive. By the end of the entry stage, the contact points decelerate and $d c / d t\left(t_{1}\right)=0$. During the exit stage, $t_{1}<t<t_{2}$, the contact region contracts with $d c / d t<0$ and $c\left(t_{2}\right)=0$. The function $c(t)$ is determined during the entry stage with the help of the Wagner condition [16]. This condition requires that the elevation of the free surface at the contact points, $\eta[ \pm c(t), t]$, is equal to the vertical position of the body at these points, $y_{b}[ \pm c(t), t]$. During the exit stage the function $c(t)$ is determined by using the condition that the speed of the contact point $d c / d t$ is proportional to the local velocity of the flow, $\varphi_{x}(c(t), 0, t)$, along the body section at this contact point (see [5]).

The boundary condition (4) is the linearized dynamic boundary condition, $p=0$, on the free surface. This condition can be integrated in time during the entry stage subject to the initial condition, $\varphi\left(x, y, t_{0}\right)=0$, giving $\varphi(x, 0, t)=0$, where $|x|>c(t)$. During the exit stage, we use the free-surface condition in the form (4). The hydrodynamic force $F(t)$ during both stages is calculated by the formula

$$
F(t)=\int_{-c(t)}^{c(t)} p(x, 0, t) d x, \quad p(x, 0, t)=-\rho \varphi_{t}(x, 0, t)
$$

The theoretical prediction of the force $F(t)$ during the entry stage can be significantly improved by using the nonlinear Bernoulli equation (2) in combination with the linearized 
hydrodynamic model (3)-(6) (see [9] and [8] for more details). However, the present analysis is focused on the exit stage. This is why we do not use the more developed entry model from [9] in this paper. Some corrections to the linear model (3)-(7) will be introduced in Section 7.

The force $F(t)$ given by (7) corresponds to the vertical force acting on the section of the $3 \mathrm{D}$ body, which is at the distance $V t$ from the leading edge of the body. The total vertical force $F_{v}$ acting on the $3 \mathrm{D}$ body is obtained by integration of the sectional forces along the body (see Figure 1)

$$
F_{v}=\int_{-V t_{2}}^{-V t_{0}} F(t) d(-V t)=V \int_{t_{0}}^{t_{2}} F(t) d t
$$

The integral in (8) is the integral of the force with respect to time and is known as the impulse $J$. The total impulse $J$ can be decomposed into the impulse $J_{i}$ during the entry stage,

$$
J_{i}=\int_{t_{0}}^{t_{1}} F(t) d t
$$

and the impulse $J_{e}$ during the exit stage,

$$
J_{e}=\int_{t_{1}}^{t_{2}} F(t) d t
$$

where $J=J_{i}+J_{e}$. Within the Wagner model of water entry, it will be shown in Section 4 that $J_{i}=0$ for any shape of the 3D body. The torque $M$ acting on the $3 \mathrm{D}$ body is calculated with respect to the section $z=-V t_{1}$, which separates the sections in entry, $-V t_{1}<z<-V t_{0}$, and the sections in exit, $-V t_{2}<z<-V t_{1}$ (see Figure 1). The torque is positive if it rotates the body in the anti-clockwise direction. The torque is calculated by using the sectional forces $(7)$, where $z=-V t$,

$$
M=\int_{-V t_{2}}^{-V t_{0}} F(-z / V)\left(z-z_{1}\right) d z=V^{2} \int_{t_{0}}^{t_{2}} F(t)\left(t_{1}-t\right) d t .
$$

The total torque can be decomposed as $M=M_{i}+M_{e}$, where

$$
M_{i}=V^{2} \int_{t_{0}}^{t_{1}} F(t)\left(t_{1}-t\right) d t, \quad M_{e}=V^{2} \int_{t_{1}}^{t_{2}} F(t)\left(t_{1}-t\right) d t
$$

$M_{i}$ is evaluated by using the force $F(t)$ provided by the Wagner model (see section 3) and $M_{e}$ is the contribution to the torque from the sections which are in exit from the water.

\section{Computational Fluid Dynamics Method}

The exit models presented in this paper are evaluated by comparing to fully nonlinear

numerical results computed using the OpenFOAM open-source CFD library. A custom 
CFD solver is used that numerically solves the incompressible Navier-Stokes equations. The air and water are considered as a single-fluid with spatially varying mechanical properties of density and viscosity. The governing equations are solved using the finite-volume method in the Arbitrary Eulerian-Lagrangian (ALE) form for moving and deforming meshes. The body force due to gravity is included and surface tension is neglected.

The governing equations are solved in the earth-fixed (stationary) coordinate system. In other words the body moves relative to the coordinate system in which the unknowns are expressed. Three cases are studied in this paper: the motion of a parabolic contour (2D), the expansion and contraction of a circular body (2D), and the translation of an ellipsoid (3D). The parabola and ellipsoid move rigidly so that the mesh moves as a solid. The expanding and contracting cylinder requires that the mesh deforms during the simulation. For the deforming mesh case, the mesh motion is governed by the Laplacian of the mesh displacement.

The interface between the air and water is tracked using the Volume-of-Fluid method. A special interface compression algorithm is used to ensure that the interface remains sharp throughout the entry and exit stages. The volume-fraction field is $\alpha$. The algorithm adaptively determines the compression coefficient depending on the relative velocity between the fluid and the mesh. The compression coefficient is calculated as

$$
C_{\alpha}=\max \left(-\frac{\mathbf{u}_{r} \cdot \nabla \alpha}{\left|\mathbf{u}_{r}\right||\nabla \alpha|+\epsilon}, 0\right)
$$

where $\mathbf{u}_{r}$ is the relative velocity between the fluid and the mesh, $\mathbf{u}-\mathbf{u}_{\text {mesh }}$, and $\epsilon$ is a small number $10^{-8}$ to ensure that the denominator does not go to zero. Additional details about the volume-of-fluid implementation in OpenFOAM and the adaptive compression can be found in $[21,13]$. The water properties are density of $1000 \mathrm{~kg} / \mathrm{m}^{3}$ and kinematic viscosity of $1 \times 10^{-6} \mathrm{~m}^{2} / \mathrm{s}$. The air properties are density of $1.5 \mathrm{~kg} / \mathrm{m}^{3}$ and kinematic viscosity of $1.5 \times 10^{-5} \mathrm{~m}^{2} / \mathrm{s}$.

The interface between the air and water is tracked using the Volume-of-Fluid method. A special interface compression algorithm is used to ensure that the interface remains sharp throughout the entry and exit stages [13]. The water properties are density of $1000 \mathrm{~kg} / \mathrm{m}^{3}$ and kinematic viscosity of $1 \times 10^{-6} \mathrm{~m}^{2} / \mathrm{s}$. The air properties are density of $1.5 \mathrm{~kg} / \mathrm{m}^{3}$ and kinematic viscosity of $1.5 \times 10^{-5} \mathrm{~m}^{2} / \mathrm{s}$.

The discretization of the fluid domain is performed using the semi-automatic mesh generation tool snappyHexMesh. Boundary layer prisms are used on the body to enhance the accuracy in the computation of the gradient of the unknowns in the direction normal to the body contour. Refinement is used in regions where the air-water interface passes. A mesh refinement study is conducted for each case, and results on the fine mesh are presented herein. The fine grid for the parabolic contour simulations uses 1,013,760 cells, and the cell size in the region where the interface passes is $6.3 \times 10^{-4} B$, where $B$ is a halfwidth of the contour. The fine grid for the expanding cylinder contains 1,635,109 cells, and the smallest cell size is $1.5 \times 10^{-4} R_{0}$, where $R_{0}$ is the initial radius of the cylinder. The ellipsoid simulations use a grid that contains more than 7.5 million cells, and the cell size in the region where the body intersects the air-water interface is $1.5 \times 10^{-4} L$, where $L$ is the largest dimension of the ellipsoid.

The boundary conditions for the flow variables on the body are zero-normal gradient for the volume fraction and dynamic pressure, and no-slip for velocity. The conditions on the far-field or extent of the domain, with the exception of the horizontal top portion and symmetry plane, are zero-normal gradient for the volume fraction and dynamic pressure, 
and zero for the velocity. On the top of the domain the conditions are zero-normal gradient for the velocity, and zero for the volume fraction and the dynamic pressure.

\section{Water-entry problem for a body of varying shape}

The hydrodynamic force $F(t)$ acting on a two-dimensional symmetric body, which enters water and at the same time changes its shape, was derived in [17] (see eq.(6)):

$$
F(t)=2 \rho \frac{\mathrm{d}^{2}}{\mathrm{~d} t^{2}}\left(c^{2} \int_{0}^{\pi / 2} y_{b}(c(t) \sin \theta, t) \sin ^{2} \theta d \theta\right)
$$

where the function $c(t)$ is the solution of the equation

$$
\int_{0}^{\pi / 2} y_{b}(c(t) \sin \theta, t) d \theta=0
$$

Equations (13) and (14) yield

$$
F(t)=-2 \rho \frac{\mathrm{d}}{\mathrm{d} t}\left[c^{2}(t) \int_{0}^{\pi / 2} y_{b, t}(c(t) \sin \theta, t) \cos ^{2} \theta d \theta\right]
$$

Equations (9) and (15) provide that the impulse $J_{i}$ during the entry stage,

$$
\int_{t_{0}}^{t_{1}} F(t) d t=-2 \rho c^{2}\left(t_{1}\right) \int_{0}^{\pi / 2} y_{b, t}\left(c\left(t_{1}\right) \sin \theta, t_{1}\right) \cos ^{2} \theta d \theta
$$

is equal to zero if the contour stops expanding at the end of the entry stage, $y_{b, t}\left(x, t_{1}\right)=0$, where $|x|<c\left(t_{1}\right)$ and $(d c / d t)\left(t_{1}\right)=0$. By using the fact that the time $t$ is proportional to the longitudinal coordinate $z$ of a section in the front part of the three-dimensional body, we can conclude that the contribution of the body sections in entry to the total vertical force (8) acting at the 3D body is zero. During the exit stage negative forces should be expected. This suggests that the total vertical force acting on a 3D body moving horizontally at a constant penetration depth is directed into the water forcing the body to sink. This results is valid for any shape of the body.

The torque $M_{i}$ given by (12) is calculated by using (13),

$$
M_{i}=2 \rho V^{2} \int_{t_{0}}^{t_{1}}\left(t_{1}-t\right) \frac{d^{2} Y(t)}{d t^{2}} d t=2 \rho V^{2}\left[\left.\left(t_{1}-t\right) \frac{d Y}{d t}\right|_{t_{0}} ^{t_{1}}+\int_{t_{0}}^{t_{1}} \frac{d Y}{d t}(t) d t\right]=2 \rho V^{2} Y\left(t_{1}\right)
$$

where

$$
Y(t)=c^{2}(t) \int_{0}^{\pi / 2} y_{b}(c(t) \sin \theta, t) \sin ^{2} \theta d \theta
$$


and $Y\left(t_{0}\right)=0, \frac{d Y}{d t}\left(t_{0}\right)=0$, which follow from (17) and (14). Equations (16), (17) and (14) show that the torque $M_{i}$ depends only on the shape of the body section at distance $V t_{1}$ from the body nose, where $t_{1}$ and $c\left(t_{1}\right)$ are determined by (14) and the equation

$$
\int_{0}^{\pi / 2} y_{b, t}\left(c\left(t_{1}\right) \sin \theta, t_{1}\right) d \theta=0,
$$

which follows from (14) and the equation $(d c / d t)\left(t_{1}\right)=0$.

The sign of the function $Y(t)$ at $t=t_{1}$ is not clear. We evaluate $Y(t)$ for a parabolic contour $y_{b}(x, t)=x^{2} /(2 R(t))+h(t)$ which penetrates the liquid with speed $\dot{h}(t)$ and changes its radius of the curvature, $R(t)$, with time. Here $h\left(t_{0}\right)=0, h(t)<0$ where $t>t_{0}$, and $\dot{h}(t) \leq 0$ where $t_{0}<t<t_{1}$. The functions $h(t)$ and $R(t)$ are assumed given. Equation (14) gives for the parabolic contour

$$
\frac{c^{2}}{2 R(t)} \int_{0}^{\pi / 2} \sin ^{2} \theta d \theta+\frac{\pi}{2} h(t)=0
$$

and

$$
c^{2}(t)=-4 h(t) R(t) .
$$

The equation $\dot{c}\left(t_{1}\right)=0$ with respect to $t_{1}$ provides

$$
\dot{h}\left(t_{1}\right) R\left(t_{1}\right)+h\left(t_{1}\right) \dot{R}\left(t_{1}\right)=0 .
$$

The function $Y(t)$ is evaluated for the parabolic contour by using (17) and (19)

$$
\begin{aligned}
Y(t)= & c^{2}(t)\left[\frac{c^{2}}{2 R(t)} \int_{0}^{\pi / 2} \sin ^{4} \theta d \theta+h(t) \int_{0}^{\pi / 2} \sin ^{2} \theta d \theta\right]= \\
& c^{2}(t)\left[\frac{3 \pi}{32} \frac{c^{2}}{R(t)}+h(t) \frac{\pi}{4}\right]=\frac{\pi}{2} R(t) h^{2}(t) .
\end{aligned}
$$

Therefore, $Y(t)$ is positive during the entry stage and the torque $M_{i}$ given by (16) rotates the body anti-clockwise. The function $Y(t)$ given by (21) for the parabolic contour and the equation (13) for the hydrodynamic force during the entry stage yield

$$
F(t)=\pi \rho \frac{\mathrm{d}^{2}}{\mathrm{~d} t^{2}}\left(R(t) h^{2}(t)\right)
$$

If the sections of the 3D body can be approximated in the contact regions, $|x|<c(t)$, by the parabolic contour with two given functions of time, $R(t)$ and $h(t)$, then the sectional forces are given by $(22)$ and the size of the contact region by (19). The torque $M_{i}$ is equal to $\pi \rho V^{2} R\left(t_{1}\right) h^{2}\left(t_{1}\right)$, where $t_{1}$ is the solution of equation (20). Equation (20) shows that, in general, the time derivatives $\dot{h}(t)$ and $\dot{R}(t)$ are not equal to zero at the end of the entry stage, when $\dot{c}\left(t_{1}\right)=0$. However, in this paper we consider only the case where both derivatives, $\dot{h}$ and $\dot{R}$ are equal to zero at $t_{1}$. In the general case, without approximation of the body sections by parabolic contour of varying curvature, we will assume that $y_{b, t}\left(x, t_{1}\right)=0$ where $|x|<c\left(t_{1}\right)$. In this case, the entry and exit stages are 
separated in time one from another and the exit stage starts at $t=t_{1}$ with $\varphi\left(x, y, t_{1}\right)=0$ in the flow region (see the problem (3)-(6)). However, the size of the contact region at the beginning of the exit stage is that at the end of the entry stage and the free surface at $t=t_{1}$ is not horizontal.

To explain how the linearised exit model can be applied to the $2 \mathrm{D}+\mathrm{T}$ problem under consideration in this study, we consider the problem of water exit on its own with both the liquid and the body being initially, $t=t_{1}$, at rest.

\section{Water exit of a rigid body}

A two-dimensional problem of a symmetric rigid body with small deadrise angle, which is lifted from the liquid surface with a prescribed acceleration, is considered within the linearized exit model [5]. The liquid is of infinite depth, inviscid and incompressible. Initially, $t=t_{1}$, the liquid is at rest. The free surface of the liquid is flat and horizontal. The body starts to move suddenly upwards from the liquid with a prescribed acceleration $\ddot{h}(t)$ which varies in time. Gravity and surface tension effects are not included in the model. Boundary conditions on the liquid surface are linearized and imposed on the equilibrium position of the liquid surface. The hydrodynamic pressure is continuous at the periphery of the wetted area which shrinks monotonically with time. The unknown size of the wetted area is determined by the condition that the speed of the contact points is proportional to the local velocity of the flow. Hydrodynamic forces acting on a lifting body are determined within the proposed linearized model and compared with the numerical results obtained by solving the Navier-Stokes equations. It is shown that the linearized water-exit model accurately predicts the hydrodynamic loads.

A linearized model of exit was suggested in [5] and applied successfully to the problems of exit with constant acceleration. In this model, the flow is assumed potential and linear. The shape of the wetted part of the body is simplified by using the so-called "flat-plate approximation". Only inertia forces are included in this exit model. Viscous effects are taken into account through the equation for the velocity of the contact points, which is assumed to be proportional to the local speed of the flow at these points.

Within the present model of water exit, the pressure $p(x, y, t)$ is given by the linearized Bernoulli equation, $p(x, y, t)=-\rho \varphi_{t}(x, y, t)$, where the velocity potential $\varphi(x, y, t)$ is a solution of the boundary-value problem (3)-(6) with $y_{b, t}(x, t)=\dot{h}(t)$ and the function $c(t)$ calculated by using the equation

$$
\frac{\mathrm{d} c}{\mathrm{~d} t}=\gamma \varphi_{x}(c(t), 0, t), \quad c\left(t_{1}\right)=c_{0},
$$

where $c_{0}$ is given. The coefficient $\gamma$ in (23) is undetermined in the present model and is chosen by using the numerical results from [10]. It was found that the theoretical predictions with $\gamma=2$ well correspond to all available numerical results. Eq. (23) assumes that the relation between the speed of the contact point $\dot{c}(t)$ and the local tangential velocity of the flow is linear with the coefficient $\gamma$ being dependent, in general, on the physical characteristics of both the liquid and the body surface, such as wettability of the body surface and viscosity of the liquid.

The estimates obtained in [5] provide that the hydrodynamic problem can be linearized

if $a T^{2} / c_{0} \ll 1$, where $a$ is the scale of the vertical acceleration of the body cross sections and $T$ is the time-scale. The surface tension and the liquid viscosity can be neglected if 
$c_{0}>3 \mathrm{~mm}$ for water at $20^{\circ} \mathrm{C}$. The gravity can be neglected if $\mathrm{g} / \mathrm{a} \ll 1$ and there is no cavitation in the wetted area during water exit if $c_{0} a<98 \mathrm{~m}^{2} \mathrm{~s}^{-2}$. For a 3D body moving along the water surface at speed $V$ with the body length $L_{b}$, characteristic size of the body sections $L_{c}$ and the penetration depth $h_{0}$ we obtain

$$
a T^{2}=O\left(h_{0}\right), \quad c_{0}=O\left(L_{c}\right), \quad T=\frac{L_{b}}{V}, \quad \frac{a T^{2}}{c_{0}}=O\left(\frac{h_{0}}{L_{c}}\right) .
$$

Therefore, the linearized model of water exit is justified if the penetration depth $h_{0}$ is smaller than the size $L_{c}$ of the body cross sections. The estimates above yield $a=$ $O\left(h_{0} V^{2} / L_{b}^{2}\right)$ which is much greater than the gravitational acceleration only for large Froude number, $V^{2} /\left(g L_{b}\right) \gg L_{b} / h_{0}$. For example, for $L_{b}=10 \mathrm{~m}, h_{0}=20 \mathrm{~cm}$ we find $V>70 \mathrm{~m} / \mathrm{s}$. For $V>100 \mathrm{~m} / \mathrm{s}$ we calculate $T=0.1 \mathrm{~s}, a \sim 20 \mathrm{~m} / \mathrm{s}^{2}$ and $c_{0} a<20 \mathrm{~m}^{2} / \mathrm{s}^{2}$. Thus, the gravity can be neglected only for a very high speed of the body. However, if the speed of the body is too high, then cavitation may start in the contact region at the end of the entry stage and the present linearized model is no longer appropriate.

Note that the linearized pressure also satisfies the Laplace equation in $y<0$, is equal to zero on the free surface, $y=0, \quad|x|>c(t)$, and $p_{y}=-\rho \ddot{h}(t)$ in the contact region, $y=0, \quad|x|<c(t)$. The latter condition has been obtained by differentiating the body boundary condition (5) in time.

The solution of the boundary problem with respect to the pressure provides (see [5])

$$
p(x, 0, t)=-\rho \ddot{h}(t) \sqrt{c^{2}(t)-x^{2}} \quad(|x|<c(t)), \quad F(t)=\int_{-c(t)}^{c(t)} p(x, 0, t) \mathrm{d} x=-m_{a} \ddot{h}(t),
$$

where $m_{a}=0.5 \pi \rho c^{2}(t)$ is the added mass of the equivalent flat plate. It is seen that the hydrodynamic force can be evaluated if the size of the wetted area, which is described by the function $c(t)$, is known.

The velocity potential and the velocity of the flow along the body surface are given by

$$
\varphi(x, 0, t)=\int_{t_{1}}^{t} \ddot{h}(\tau) \sqrt{c^{2}(\tau)-x^{2}} d \tau \quad \varphi_{x}(x, 0, t)=-x \int_{t_{1}}^{t} \frac{\ddot{h}(\tau) d \tau}{\sqrt{c^{2}(\tau)-x^{2}}} .
$$

Equations (23) and (25) yield the following integro-differential equation for the function $c(t)$

$$
\frac{\mathrm{d} c}{\mathrm{~d} t}=-\gamma c(t) \int_{t_{1}}^{t} \frac{\ddot{h}(\tau) \mathrm{d} \tau}{\sqrt{c^{2}(\tau)-c^{2}(t)}} .
$$

Equation (26) can be solved numerically for a given function $h(t)$ but we could not find a stable algorithm to discretize equation (26). Below we transform equation (26) to a system of two equations with respect to two new unknown functions, as it was suggested in [5], and discretize these two equations with account for the initial asymptotic behaviour of its solution.

To solve equation (26) with a given function $h(t)$ and the initial condition $c\left(t_{1}\right)=c_{0}$, we introduce new non-dimensional variables $\alpha$ and $\sigma$ such that $c^{2}(t)=c_{0}^{2}(1-\sigma), c^{2}(\tau)=$ $c_{0}^{2}(1-\alpha)$, where $\alpha$ and $\sigma$ are equal to zero when $t=t_{1}$ and $\tau=t_{1}$, correspondingly, and 
$\alpha=\sigma$ at $\tau=t$ (see [5] for details). A new unknown function $f(\sigma)$ is introduced by the equation

$$
\ddot{h}=f(\sigma) \frac{\mathrm{d} c^{2}}{\mathrm{~d} t} .
$$

Then equation (26) leads to two equations with respect to $f(\sigma)$ and $t(\sigma), 0<\sigma<1$,

$$
\dot{h}(t)=-c_{0}^{2} \int_{0}^{\sigma} f(\alpha) \mathrm{d} \alpha, \quad \ddot{h}(t)=2 \gamma c_{0}^{3}(1-\sigma) f(\sigma) \int_{0}^{\sigma} \frac{f(\alpha) \mathrm{d} \alpha}{\sqrt{\sigma-\alpha}} .
$$

The system (27) is not ready yet for its numerical analysis. Indeed, if $\ddot{h}(0) \neq 0$, then $f(\sigma) \rightarrow \infty$ as $\sigma \rightarrow 0$ which follows from the second equation of the system. To prove this asymptotic behaviour, let us assume that $f(\sigma)$ tends to a constant as $\sigma \rightarrow 0$. Then the integral in the second equation tends to zero and the right-hand side of this equation tends to zero but the left-hand side, $\ddot{h}(t)$, is not zero as $t \rightarrow t_{1}$. To cover a general case of the body motion, we assume that the body displacement $h(t)$ behaves as $h(t) \sim A\left(t-t_{1}\right)^{m}$ when $t \rightarrow t_{1}$. Here $A>0$ and $m>1$. The latter inequality implies that $\dot{h}(0)=0$. The asymptotic behaviour of the function $f(\sigma)$ as $\sigma \rightarrow 0$ is sought in the form $f(\sigma) \sim-q_{o} \sigma^{-k}$. The system (27) provides as $\sigma \rightarrow 0$ and $t(\sigma) \rightarrow t_{1}$ :

$$
\begin{gathered}
A m\left(t(\sigma)-t_{1}\right)^{m-1} \sim q_{0} c_{0}^{2} \int_{0}^{\sigma} \alpha^{-k} d \alpha, \\
A m(m-1)\left(t(\sigma)-t_{1}\right)^{m-2} \sim 2 \gamma q_{0}^{2} c_{0}^{3} \sigma^{-k} \int_{0}^{\sigma} \frac{\alpha^{-k} d \alpha}{\sqrt{\sigma-\alpha}} .
\end{gathered}
$$

The integrals in (28) and (29) are evaluated as

$$
\int_{0}^{\sigma} \alpha^{-k} d \alpha=\frac{\sigma^{1-k}}{1-k}, \quad \int_{0}^{\sigma} \frac{\alpha^{-k} d \alpha}{\sqrt{\sigma-\alpha}}=\sigma^{1 / 2-k} \chi_{k}, \quad \chi_{k}=\int_{0}^{1} \frac{u^{-k} d u}{\sqrt{1-u}} .
$$

The right-hand side in (29) is of the order of $O\left(\sigma^{1 / 2-2 k}\right)$ as $\sigma \rightarrow 0$. Therefore,

$$
t(\sigma)-t_{1}=O\left(\sigma^{\frac{1 / 2-2 k}{m-2}}\right)
$$

and the asymptotic estimate (28) gives

$$
q_{0} c_{0}^{2} \frac{\sigma^{1-k}}{1-k}=O\left(\sigma^{\frac{(1 / 2-2 k)(m-1)}{m-2}}\right) .
$$

The order of the right-hand side and left-hand side in (31) as $\sigma \rightarrow 0$ are equal if

$$
1-k=\frac{m-1}{m-2}\left(\frac{1}{2}-2 k\right)
$$

which yields the formula for the power $k$

$$
k=\frac{3-m}{2 m} .
$$


If the contour starts to exit water with $\ddot{h}(0) \neq 0$, then $m=2$ and $k=1 / 4$, which is the result from [5]. If $m \geq 3$, then the function $f(\sigma)$ is regular at $\sigma=0$.

The definition of the variable $\sigma, c^{2}(t)=c_{0}^{2}(1-\sigma)$, and equations (28), (32) provides the asymptotic behaviour of the coordinate of the contact point as $t \rightarrow t_{1}$,

$$
\begin{gathered}
c(t)=c_{0} \sqrt{1-\sigma}=c_{0}\left(1-\frac{1}{2} \sigma+O\left(\sigma^{2}\right)\right)= \\
=c_{0}-\frac{1}{2} c_{0}\left(\frac{A m(1-k)}{c_{0}^{2} q_{0}}\right)^{\frac{1}{1-k}}\left(t-t_{1}\right)^{\frac{m-1}{1-k}}+O\left(\left|t-t_{1}\right|^{2 \frac{m-1}{1-k}}\right),
\end{gathered}
$$

where $(m-1) /(1-k)=2 m / 3$. It is seen that the initial velocity of the contact point, $\dot{c}\left(t_{1}\right)$, is zero if $m>3 / 2$. Eliminating $t(\sigma)-t_{1}$ from (28), (29) and using (32), we obtain the coefficient $q_{0}$

$$
q_{0}=\frac{2 m}{3 c_{0}}\left(\frac{9}{4} \frac{m-1}{m} \frac{A}{c_{0}}\right)^{1 / m}\left(2 \gamma \chi_{k}\right)^{\frac{1-m}{m}} .
$$

For a body lifted from the water surface at a constant acceleration $a$, we have $m=2$, $A=\frac{1}{2} a$ and equation (34) provides

$$
q_{0}=\left(\frac{a}{2 \gamma \chi_{1 / 4} c_{0}^{3}}\right)^{1 / 2}
$$

which is the formula from [1].

By using the obtained asymptotic behaviour of the function $f(\sigma)$ as $\sigma \rightarrow 0$, we introduce new non-dimensional function $G(\sigma)$ by

$$
f(\sigma)=-q_{0} \sigma^{-k} G(\sigma)
$$

where $G(\sigma)$ is regular at $\sigma=0$ and $G(0)=1$. Substituting (35) in (27) and introducing non-dimensional velocity $V(t)=\dot{h}(t) /\left(c_{0}^{2} q_{0}\right)$ and acceleration $W(t)=\ddot{h}(t) /\left(2 \gamma c_{0}^{3} q_{0}^{2}\right)$ of the body, we arrive at the system

$$
V(t)=\int_{0}^{\sigma} \alpha^{-k} G(\alpha) d \alpha, \quad W(t)=(1-\sigma) \sigma^{-k} G(\sigma) \int_{0}^{\sigma} G(\alpha) \frac{\alpha^{-k} d \alpha}{\sqrt{\sigma-\alpha}}
$$

in the non-dimensional variables, where $0<\sigma<1, G(0)=1$ and the functions $V(t)$ and $W(t), t>t_{1}$, are given. The system (36) is suitable for numerical analysis.

The interval $0<\sigma<1$ is divided into subintervals $\left(\sigma_{n-1}, \sigma_{n}\right)$ of length $\Delta \sigma$, where $n \geq 1, \sigma_{n}=n \Delta \sigma$ and $\sigma_{0}=0$. The function $G(\sigma)$ is linearly interpolated in each subinterval. Then the first equation of the system (36) gives

$$
\begin{gathered}
V\left(t_{n}\right)-V\left(t_{n-1}\right)=\int_{\sigma_{n-1}}^{\sigma_{n}} \alpha^{-k} G(\alpha) d \alpha \approx \\
\int_{\sigma_{n-1}}^{\sigma_{n}} \alpha^{-k}\left(G_{n-1}+\frac{G_{n}-G_{n-1}}{\Delta \sigma}\left(\alpha-\sigma_{n-1}\right)\right) d \alpha=\alpha_{n} G_{n}-\beta_{n} G_{n-1}
\end{gathered}
$$


where $G_{n}=G\left(\sigma_{n}\right), t_{n}=t\left(\sigma_{n}\right)$ and $\alpha_{n}, \beta_{n}$ are the known coefficients. We obtain

$$
G_{n}=\left(V\left(t_{n}\right)-V\left(t_{n-1}\right)+\beta_{n} G_{n-1}\right) / \alpha_{n}
$$

Equation (37) defines $G\left(\sigma_{n}\right)$ as a function of $t_{n}$ once $t_{n-1}$ and $G_{n-1}$ have been determined in the previous time step. The second equation in (36) at $\sigma=\sigma_{n}$ gives

$$
\begin{gathered}
W\left(t_{n}\right)=\left(1-\sigma_{n}\right) \sigma_{n}^{-k} G_{n} \sum_{j=1}^{n} \int_{\sigma_{j-1}}^{\sigma_{j}} G(\alpha) \frac{\alpha^{-k} d \alpha}{\sqrt{\sigma_{n}-\alpha}} \approx \\
\left(1-\sigma_{n}\right) \sigma_{n}^{-k} G_{n}\left\{\frac{G_{n}}{\Delta \sigma} \int_{\sigma_{n-1}}^{\sigma_{n}} \frac{\alpha^{-k}\left(\alpha-\sigma_{n-1}\right) d \alpha}{\sqrt{\sigma_{n}-\alpha}+P_{n}}\right\},
\end{gathered}
$$

where $P_{n}$ depends on $G_{j}$ with $1 \leq j \leq n-1$. The resulting equation is

$$
W\left(t_{n}\right)=\left(1-\sigma_{n}\right) \sigma_{n}^{-k} G_{n}\left(G_{n} A_{n}+P_{n}\right), \quad A_{n}=\sqrt{\Delta \sigma} \int_{0}^{1}\left(\sigma_{n-1}+\Delta \sigma \xi\right)^{-k} \frac{\xi d \xi}{\sqrt{1-\xi}}
$$

Substituting (37) in (38) we obtain the non-linear equation with is respect to $t_{n}$, which is solved by the bisection method.

Computations are performed for the parabolic contour $y=x^{2} /(2 R)-h_{0}+h(t)$, where $R \approx 1.4 \mathrm{~m}, h_{0}=1 \mathrm{~cm}, t_{1}=0$ and $\ddot{h}(t)=a+2 b t, a=1 \mathrm{~m} / \mathrm{s}^{2}, b= \pm 1 \mathrm{~m} / \mathrm{s}^{3}$. Initially, $t=0$, the contour is submerged at depth $h_{0}$. Both the liquid and the body are initially at rest. Here $c_{0}=\sqrt{2 R h_{0}} \approx 17 \mathrm{~cm}$. The theoretical force does not depend on the body shape but on $c_{0}$ and the body displacement $h(t)$. The size of the contact area, $c(t)$, is calculated by (37) and (38) with $\Delta \sigma=5 \cdot 10^{-3}$. The force is calculated by equation (24), which provides

$$
F\left(t_{n}\right)=-\pi \gamma \rho c_{0}^{5} q_{0}^{2}\left(1-\sigma_{n}\right) W\left(t_{n}\right) .
$$

In the present case, $m=2, A=a / 2$ and equations (32) and (34) give $k=1 / 4$ and

$$
q_{0}^{2}=\frac{a}{2 \gamma c_{0}^{3} \chi_{1 / 4}}
$$

Equations (39) and (40) show that the magnitude of the force $F(t)$ is independent of the parameter $\gamma$. Below we take $\gamma=2$ as in [5].
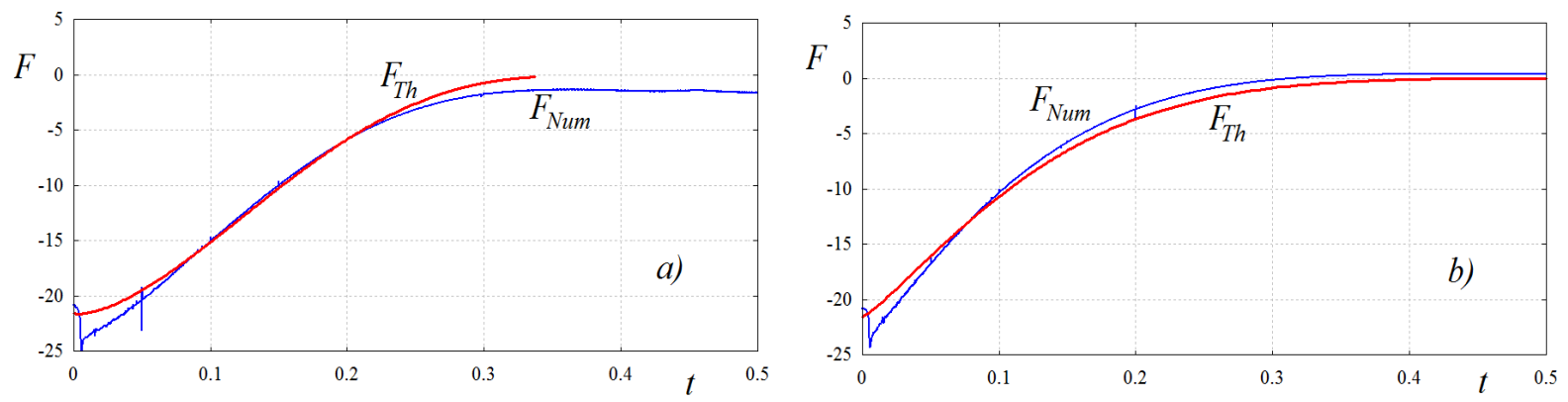

Fig 3. The dimensional force in $N / m$ acting on a half of the body as a function of time in seconds for $a=1 \mathrm{~m} / \mathrm{s}^{2}, g=0$ and $b=1 \mathrm{~m} / \mathrm{s}^{3}$ (a), $b=-1 \mathrm{~m} / \mathrm{s}^{3}$ (b). 
The problem of water exit for the same conditions is also solved numerically with a VOF-based Navier-Stokes solver from the OpenFOAM library. Gravity is not included in the computations. The results for the vertical force on the body are presented in Figure 3. It is seen that the theoretical model well describes the evolution of the hydrodynamic force. However, the model does not describe all details of the force just after the start of the body motion, and the interaction lasts longer in CFD than in the theory for $b=1 \mathrm{~m} / \mathrm{s}^{3}$.

The shape of the free surface predicted by the numerical solution for $a=1 \mathrm{~m} / \mathrm{s}^{2}$ and $b=1 \mathrm{~m} / \mathrm{s}^{3}$ at several time instants is shown in Figure 4 . The wetted part of the body surface is much larger in the numerical solution than in the linearized exit model. This explains why the numerical force lasts longer than the theoretical one (see Figure 3a). However, the distance travelled by the body by $t=0.3 \mathrm{~s}$ is $5.4 \mathrm{~cm}$ which is not small compared with the horizontal size of the contact region $c_{0}$. The linearized model of water exit is not accurate for large displacements of the lifting body. On the other hand, the forces at the end of the exit stage are rather small (see Figure 3) and can be neglected in calculations of the body dynamics but not of the flow, which is nonlinear and complicated at the end of the exit stage (see Figure 4). Note that the system (27) can be used only for motions with positive acceleration, $\ddot{h}(t)>0$. Only for such motions the function $f(\sigma)$ can be introduced. The function $f(\sigma)$ is singular as $\sigma \rightarrow 1$ (see [5] for details). The solution of the system (27) is not reliable at the end of the exit stage when $\sigma \rightarrow 1$ and $c(t) \rightarrow 0$.

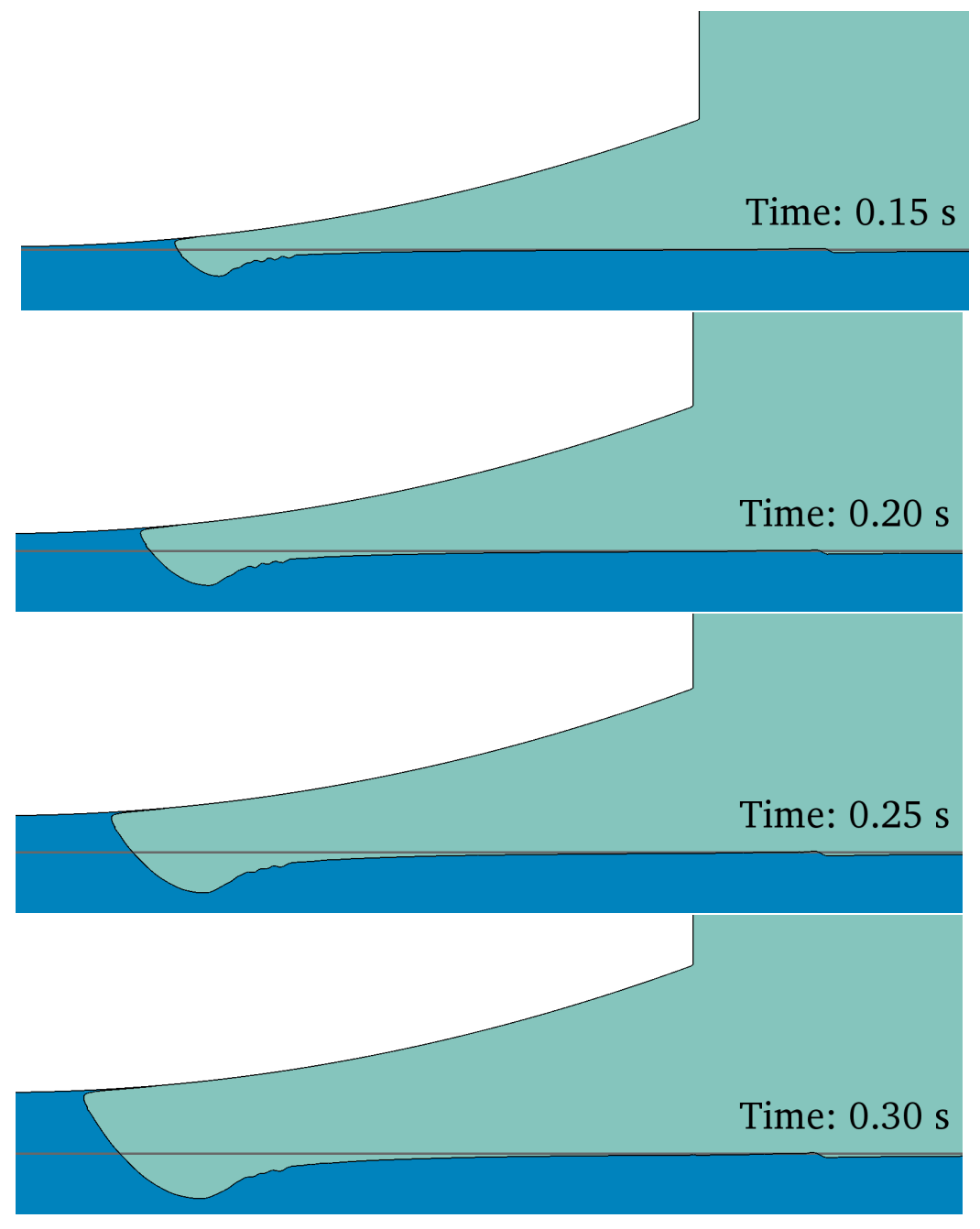




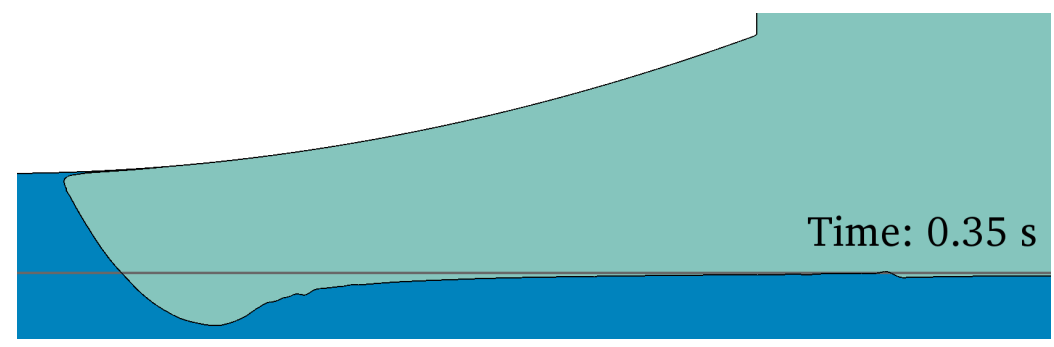

Fig 4. CFD prediction of free-surface position for the case shown in Figure 3a.

Equation (32) shows that the function $f(\sigma)$ is regular at $\sigma=0$ for $m=3$. The case with $m=3$ corresponds to the motion of a floating body with linear in time acceleration, $h(t)=a t^{3}$. In this case, $k=0$ and the system (36) can be written in the form independent of any parameters

$$
\frac{\tau^{2}}{4}=\int_{0}^{\sigma} G(\alpha) \mathrm{d} \alpha, \quad \tau=(1-\sigma) G(\sigma) \int_{0}^{\sigma} \frac{G(\alpha) \mathrm{d} \alpha}{\sqrt{\sigma-\alpha}},
$$

where $t=\tau\left(c_{0} / 48 \gamma a\right)^{\frac{1}{3}}, G(0)=1$ and the force $F(t)=F_{s c} \tau(\sigma)(\sigma-1)$ with the scale $F_{s c}=0.25 \pi \rho\left(36 a^{2} c_{0}^{7} / \gamma\right)^{\frac{1}{3}}$. The computations were performed for the same shape as in Figures 3 and 4 . The system (41) is solved by the power series method similar to that from [5]. The results are shown in Figure 5a. It was found that the difference between the numerical, $F_{\text {num }}(t)$, and theoretical, $F_{t h}(t)$, solutions can be approximated by $5.5 t$ (see Figure 5b). This implies that the added mass in the CFD results differs from the theoretical one by a constant. One could expect that this constant describes the effect of the liquid left on the lifting surface. The latter effect is not taken into account in the theoretical model.
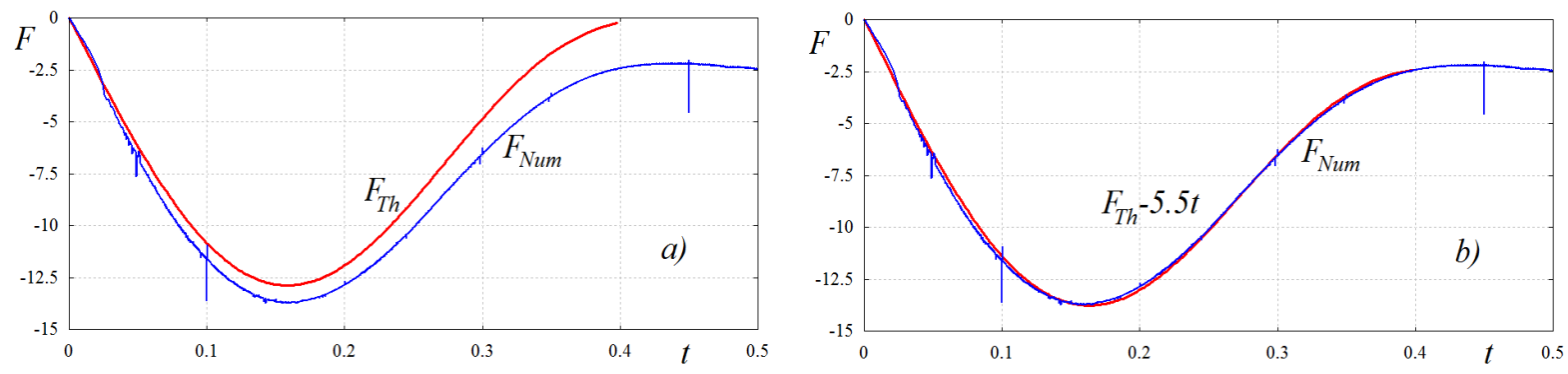

Fig 5. The dimensional force in $N / m$ acting on a half of the parabolic contour lifted from water with linear acceleration, $h(t)=a t^{3}$, where $a=1 \mathrm{~m} / \mathrm{s}^{3}$.

\section{Water exit of a body with time-varying shape}

In this section, we study the problem of water exit, (3)-(6), with the condition (23) and a given function $y_{b}(x, t)$ in the body boundary condition (5). The problem was solved in [8] by using the von Karman model [18], where the positions of the contact points, $x= \pm c(t)$, are determined as the intersection points between the moving surface of the body and the initial position of the free surface. Here we employ the linearized model of water exit generalized in Section 5. 


\subsection{Reformulation of Boundary Value Problem}

The problem (3)-(6) is reformulated with respect to the analytic function

$$
W(z, t)=\left(\varphi_{x t}-i \varphi_{y t}\right) \sqrt{z^{2}-c^{2}(t)}
$$

in the lower half-plane, $y<0$, where $z=x+i y$ and $W(z, t) \rightarrow 0$ as $z \rightarrow \infty$. On the boundary, $y=0$, we calculate

$$
W(x-i 0, t)= \begin{cases}-i \varphi_{y t} \sqrt{x^{2}-c^{2}(t)} & x>c(t), \\ -i \sqrt{c^{2}(t)-x^{2}}\left(\varphi_{x t}-i y_{b, t t}(x, t)\right) & |x|<c(t), \\ i \varphi_{y t} \sqrt{x^{2}-c^{2}(t)} & x<-c(t) .\end{cases}
$$

and

$$
\operatorname{Re}[W(x-i 0, t)]= \begin{cases}-\sqrt{c^{2}(t)-x^{2}} y_{b, t t}(x, t) & |x|<c(t), \\ 0 & |x|>c(t) .\end{cases}
$$

The Hilbert formula (which relates the real and imaginary parts of an analytic function in the lower half-plane, $y<0)$ written for the interval $|x|<c(t)$ provides

$$
\varphi_{x t}(x, 0, t)=\frac{1}{\pi \sqrt{c^{2}-x^{2}}} \mathrm{p} \cdot \mathrm{v} \cdot \int_{-c(t)}^{c(t)} y_{b, t t}(\xi, t) \frac{\sqrt{c^{2}-\xi^{2}} d \xi}{\xi-x} .
$$

The horizontal component of the flow velocity on the boundary, $y=0$, is given by

$$
\varphi_{x}(x, 0, t)=\int_{t_{1}}^{t} \varphi_{x t}(x, 0, \tau) d \tau
$$

Note that $\varphi\left(x, y, t_{1}\right)=0$ within the linearized model of water exit. Equations (23), (44) and (43) yield

$$
\frac{d c}{d t}=\gamma \varphi_{x}[c(t), 0, t]=\frac{\gamma}{\pi} \int_{t_{1}}^{t} \frac{1}{\sqrt{c^{2}(\tau)-c^{2}(t)}} \mathrm{p} \cdot \mathrm{v} \cdot \int_{-c(\tau)}^{c(\tau)} y_{b, t t}(\xi, \tau) \frac{\sqrt{c^{2}(\tau)-\xi^{2}} d \xi}{\xi-c(t)} d \tau
$$

where $c\left(t_{1}\right)=c_{0}$. This is the equation with respect to the size of the wetted part of the body surface, $c(t)$, for given $y_{b}(x, t)$ and $c_{0}$.

The integral with respect to $\xi$ in (45) is the Cauchy Principal Value integral, where $c(t)<c(\tau)$ for $t>\tau$. This integral can be transformed as

$$
\begin{gathered}
\text { p.v. } \int_{-c(\tau)}^{c(\tau)} y_{b, t t}(\xi, \tau) \frac{\sqrt{c^{2}(\tau)-\xi^{2}} d \xi}{\xi-c(t)}= \\
=\left[c^{2}(\tau)-c^{2}(t)\right] \operatorname{p.v} \cdot \int_{-c(\tau)}^{c(\tau)} y_{b, t t}(\xi, \tau) \frac{d \xi}{(\xi-c(t)) \sqrt{c^{2}(\tau)-\xi^{2}}}-\int_{-c(\tau)}^{c(\tau)} y_{b, t t}(\xi, \tau) \frac{(\xi+c(t)) d \xi}{\sqrt{c^{2}(\tau)-\xi^{2}}},
\end{gathered}
$$


where the first integral is zero if $y_{b, t t}(\xi, \tau)$ does not depend on $\xi$. In the second integral, $\xi$ in the numerator gives zero contribution because $y_{b, t t}(\xi, \tau)$ is even function of $\xi$. To solve equation (45), we introduce a new function

$$
H(\tau)=\frac{2}{\pi} \int_{0}^{c(\tau)} \frac{y_{b, t t}(\xi, \tau) d \xi}{\sqrt{c^{2}(\tau)-\xi^{2}}}
$$

a new variable $\sigma=\sigma(t)$, such that $c^{2}(t)=c_{0}^{2}(1-\sigma)$ and $\sigma\left(t_{1}\right)=0$, and a new function $f(\sigma)$ related to $H(t)$ by

$$
H(t)=f(\sigma) \frac{d c^{2}(t)}{d t}=-c_{0}^{2} f(\sigma) \frac{d \sigma}{d t}
$$

which is similar to the analysis in Section 5. Letting $c^{2}(\tau)=c_{0}^{2}(1-\alpha)$, we can write equation (45) in the form

$$
\frac{d c}{d t}=-\gamma c(t) \int_{t_{1}}^{t} \frac{H(\tau) d \tau}{\sqrt{c^{2}(\tau)-c^{2}(t)}}+\gamma S(t)=\gamma c_{0} c(t) \int_{0}^{\sigma} \frac{f(\alpha) d \alpha}{\sqrt{\sigma-\alpha}}+\gamma S(t),
$$

where

$$
S(t)=\int_{t_{1}}^{t} \sqrt{c^{2}(\tau)-c^{2}(t)}\left[\frac{1}{\pi} \int_{-c(\tau)}^{c(\tau)} \frac{y_{b, t t}(\xi, \tau)-y_{b, t t}(c(t), \tau)}{(\xi-c(t)) \sqrt{c^{2}(\tau)-\xi^{2}}} d \xi\right] d \tau .
$$

If $y_{b, t t}(x, \tau)=\ddot{h}(\tau)$ as in Section 5 , then $S(t) \equiv 0, H(t)=\ddot{h}(t)$ and equation (48) coincides with (26).

Next we divide both sides of equation (48) by $\dot{c}(t)$ and multiply by $H(t)$

$$
H(t)=\gamma c_{0} \frac{c(t)}{\dot{c}(t)} H(t) \int_{0}^{\sigma} \frac{f(\alpha) d \alpha}{\sqrt{\sigma-\alpha}}+\gamma \frac{H(t)}{\dot{c}(t)} S(t)
$$

where

$$
c_{0} \frac{c(t)}{\dot{c}(t)} H(t)=2 c_{0}^{3}(1-\sigma) f(\sigma), \quad \frac{H(t)}{\dot{c}(t)}=2 c(t) f(\sigma)
$$

Then

$$
H(t)=2 \gamma c_{0}^{3}(1-\sigma) f(\sigma) \int_{0}^{\sigma} \frac{f(\alpha) d \alpha}{\sqrt{\sigma-\alpha}}+2 \gamma c_{0} f(\sigma) S(t) \sqrt{1-\sigma} .
$$

Equation (50) has to be solved with respect to $f(\sigma)$ and $t(\sigma)$ together with the equation which follows from (47)

$$
\int_{t_{1}}^{t} H(\tau) d \tau=-c_{0}^{2} \int_{0}^{\sigma} f(\alpha) d \alpha
$$

The system (50), (51) reduces to the system (27) if the body exits water without changing its shape in time, $y_{b, t t}(x, t)=\ddot{h}(t)$. 
Once the system (50), (51) has been solved, we obtain $c=c(t)$ and can compute the hydrodynamic force $F(t)$ acting on the body of variable shape during the exit stage. Within the linearized exit model

$$
F(t)=\int_{-c(t)}^{c(t)} p(x, 0, t) d x=\rho \int_{-c(t)}^{c(t)} x \varphi_{t x}(x, 0, t) d x
$$

where $\varphi_{t x}(x, 0, t)$ in the contact region is given by (43). Substituting this formula for $\varphi_{t x}(x, 0, t)$ in $(52)$ we find

$$
\begin{gathered}
F(t)=\frac{1}{\pi} \rho \int_{-c(t)}^{c(t)} \frac{x}{\sqrt{c^{2}-x^{2}}}\left[\mathrm{p} \cdot \mathrm{v} \cdot \int_{-c(t)}^{c(t)} y_{b, t t}(\xi, t) \frac{\sqrt{c^{2}-\xi^{2}} d \xi}{\xi-x}\right] d x= \\
\frac{1}{\pi} \rho \int_{-c(t)}^{c(t)} y_{b, t t}(\xi, t) \sqrt{c^{2}-\xi^{2}}\left[\mathrm{p} \cdot \mathrm{v} \cdot \int_{-c(t)}^{c(t)} \frac{x d x}{\sqrt{c^{2}-x^{2}}(\xi-x)}\right] d \xi,
\end{gathered}
$$

which gives

$$
F(t)=-2 \rho c^{2}(t) \int_{0}^{\pi / 2} y_{b, t t}(c(t) \sin \theta, t) \cos ^{2} \theta d \theta .
$$

If the body shape does not change in time, then $y_{b, t t}(c(t) \sin \theta, t)=\ddot{h}(t), \int_{0}^{\pi / 2} \cos ^{2} \theta d \theta=$ $\pi / 4$ and (53) gives

$$
F(t)=-\frac{\pi}{2} \rho c^{2}(t) \ddot{h}(t)
$$

which is equation (24) from Section 5.

\subsection{Solution of Boundary Value Problem}

The system (50), (51) with respect to two unknown functions, $f(\sigma)$ and $t(\sigma)$ is solved numerically by a $\sigma$-marching procedure similar to that described in Section 5 . Note that the integral in (46) depends on $t(\alpha)$ through $\tau$ in the numerator and $\alpha$ through $c(\tau)=c_{0} \sqrt{1-\alpha}$. It is convenient in the following to change $H(t)$ in (50) to $H(\sigma, t(\sigma))$ showing explicitly the functions to be determined. Then equation (50) takes the form

$$
\begin{gathered}
H(\sigma, t(\sigma))=2 \gamma c_{0}^{3}(1-\sigma) f(\sigma) \int_{0}^{\sigma} \frac{f(\sigma)}{\sqrt{\sigma-\alpha}} R(\sigma, \alpha, t(\alpha)) d \alpha, \\
H(\sigma, t(\sigma))=\frac{2}{\pi} \int_{0}^{\pi / 2} y_{b, t t}\left(c_{0} \sqrt{1-\sigma} \sin \theta, t(\sigma)\right) d \theta, \\
R(\sigma, \alpha, t(\sigma), t(\alpha))=1-2(\sigma-\alpha) \frac{L(\sigma, \alpha, t(\alpha))}{H(\alpha, t(\alpha))},
\end{gathered}
$$




$$
L(\sigma, \alpha, t(\alpha))=\int_{0}^{\pi / 2} \frac{y_{b, t t}\left(c_{0} \sqrt{1-\alpha} \sin \theta, t(\alpha)\right)-y_{b, t t}\left(c_{0} \sqrt{1-\sigma}, t(\alpha)\right)}{(1-\alpha) \sin ^{2} \theta-(1-\sigma)} d \theta .
$$

For a rigid body with $y_{b, t t}(x, t)=\ddot{h}(t)$ we have $L(\sigma, \alpha, t(\alpha)) \equiv 0, R \equiv 1, H(\sigma, t(\sigma))=$ $\ddot{h}[t(\sigma)]$. Correspondingly, equation (51) takes the form

$$
\int_{t(0)}^{t(\sigma)} H(\sigma(\tau), \tau) d \tau=-c_{0}^{2} \int_{0}^{\sigma} f(\alpha) d \alpha
$$

and equation (54) is reduced to the second equation in (27).

For a parabolic contour with a time-dependent curvature, $y_{b}(x, t)=x^{2} B(t)-h_{0}+h(t)$, calculations provide

$$
\begin{gathered}
y_{b, t t}(x, t)=x^{2} \ddot{B}(t)+\ddot{h}(t), \\
H(\sigma, t(\sigma))=\frac{c_{0}^{2}}{2}(1-\sigma) \ddot{B}(t(\sigma))+\ddot{h}(t(\sigma)), \quad L(\sigma, \alpha, t(\alpha))=\frac{\pi}{2} c_{0}^{2} \ddot{B}(t(\alpha)) .
\end{gathered}
$$

The system (54), (55) is studied here only for the case $H\left(0, t_{1}\right)=a>0$. The unknown function $f(\sigma)$ behaves as $f(\sigma)=-q_{0} \sigma^{-1 / 4}$ when $\sigma \rightarrow 0$ (see Section 5), where $q_{0}=$ $\left(a /\left[2 \gamma c_{0}^{3} \chi_{1 / 4}\right]\right)^{1 / 2}$. We introduce the new unknown function $G(\sigma)$ by $(35), G(0)=1$, and write the system $(54),(55)$ in the form suitable for its numerical analysis

$$
\begin{aligned}
H(\sigma, t(\sigma))= & w(1-\sigma) \sigma^{-1 / 4} G(\sigma) \int_{0}^{\sigma} \frac{\alpha^{-1 / 4} G(\alpha)}{\sqrt{\sigma-\alpha}} R(\sigma, \alpha, t(\alpha)) d \alpha, \\
& \int_{t(0)}^{t(\sigma)} H(\sigma(\tau), \tau) d \tau=v \int_{0}^{\sigma} \alpha^{-1 / 4} G(\alpha) d \alpha
\end{aligned}
$$

where $w=2 \gamma q_{0}^{2} c_{0}^{3}=a / \chi_{1 / 4}$ is the scaled initial acceleration of the body and $v=c_{0}^{2} q_{0}$ is a characteristic velocity.

The system (56), (57) is solved by the collocation method similar to that from Section 5. Within this method the equations are satisfied at $\sigma=\sigma_{j}, j=1, \ldots N$, where $\Delta \sigma=1 /(N+1)$. Then $\sigma_{N}=1-\Delta \sigma$. The end point $\sigma=1$, where the contact region is a single point, is not included in computations. The time instant corresponding to $\sigma_{j}$ is denoted by $t_{j}, t_{j}=t\left(\sigma_{j}\right)$. Equations (56) and (57) provide

$$
\begin{aligned}
H\left(\sigma_{j}, t_{j}\right)=w & \left(1-\sigma_{j}\right) \sigma_{j}^{-1 / 4} G_{j}\left[\int_{\sigma_{j-1}}^{\sigma_{j}} \frac{\alpha^{-1 / 4} G(\alpha)}{\sqrt{\sigma_{j}-\alpha}} R\left(\sigma_{j}, \alpha, t(\alpha)\right) d \alpha+\right. \\
& \left.+\int_{0}^{\sigma_{j-1}} \frac{\alpha^{-1 / 4} G(\alpha)}{\sqrt{\sigma_{j}-\alpha}} R\left(\sigma_{j}, \alpha, t(\alpha)\right) d \alpha,\right] \\
& \int_{t_{j-1}}^{t_{j}} H(\sigma(\tau), \tau) d \tau=v \int_{\sigma_{j-1}}^{\sigma_{j}} \alpha^{-1 / 4} G(\alpha) d \alpha
\end{aligned}
$$


where $G_{j}=G\left(\sigma_{j}\right), H_{j}=H\left(\sigma_{j}, t_{j}\right), R_{j i}=R\left(\sigma_{j}, \sigma_{i}, t_{i}\right), 1 \leq j \leq N, i \leq j, R_{j j}=1$. The function $H(\sigma(\tau), \tau)$ in the interval $t_{j-1}<\tau<t_{j}$ and the function $G(\alpha)$ in the interval $\left(\sigma_{j-1}, \sigma_{j}\right)$ are approximated by linear functions. Then equation (59) yields

$$
\frac{1}{2}\left(H_{j}+H_{j-1}\right)\left(t_{j}-t_{j-1}\right) \approx v\left(G_{j-1} a_{j}+\left[G_{j}-G_{j-1}\right] b_{j}\right),
$$

which gives $G_{j}$ as a function of $t_{j}$,

$$
G_{j}=\frac{1}{2 b_{j} v}\left(H_{j}+H_{j-1}\right)\left(t_{j}-t_{j-1}\right)+G_{j-1}\left(1-\frac{a_{j}}{b_{j}}\right)
$$

where $H_{j-1}, G_{j-1}, t_{j-1}$ are assumed known from the previous step of calculations, and

$$
\begin{gathered}
a_{j}=\int_{\sigma_{j-1}}^{\sigma_{j}} \alpha^{-1 / 4} d \alpha=\frac{4}{3}\left(\sigma_{j}^{3 / 4}-\sigma_{j-1}^{3 / 4}\right), \\
b_{j}=\frac{1}{\Delta \sigma} \int_{\sigma_{j-1}}^{\sigma_{j}} \alpha^{-1 / 4}\left(\alpha-\sigma_{j-1}\right) d \alpha=\frac{4}{3} \sigma_{j}^{3 / 4}-\frac{16}{21} \frac{\sigma_{j}^{7 / 4}-\sigma_{j-1}^{7 / 4}}{\Delta \sigma} .
\end{gathered}
$$

In equation (58), the integrals are evaluated approximately by using linear approximations of the product $G(\alpha) R\left(\sigma_{j}, \alpha, t(\alpha)\right)$ in the intervals $\left(\sigma_{i-1}, \sigma_{i}\right)$, where $i \leq j$,

$$
G(\alpha) R\left(\sigma_{j}, \alpha, t(\alpha)\right)=G_{i-1} R_{j, i-1}+\frac{G_{i} R_{j, i}-G_{i-1} R_{j, i-1}}{\Delta \sigma}\left(\alpha-\sigma_{i-1}\right) .
$$

Then equation (58) can be approximated by

$$
H_{j}=w\left(1-\sigma_{j}\right) \sigma_{j}^{-1 / 4} G_{j}\left[G_{j} A_{j}+Q_{j}\right]
$$

where the coefficients $A_{j}$ were introduced in (38) and $Q_{j}$ depends on $G_{i}$ with $1 \leq i \leq j-1$. The resulting system (60), (61) is similar to the system (37), (38) from Section 5. Substituting $G_{j}$ from (60) in (61), we arrive at the equation with respect to $t_{j}$, which is solved by the bisection method.

\subsection{Analysis of Expanding and Contracting Cylinder}

The derived exit model (53)-(55) is applied to the problem of expanding and contracting circular cylinder [8] with the time-varying shape of the body given by

$$
y_{b}(x, t)=R_{0}-\sqrt{R^{2}(t)-x^{2}}, \quad R(t)=R_{0}+R_{1} t+R_{2} t^{2},
$$

where $t_{0}=0$ (see Figure 2) and $R_{0}$ is the initial radius of the cylinder. Initially the liquid is at rest and occupies the lower half-plane, $y<0$, with the horizontal free surface at $y=0$. The cylinder touches the free surface at a single point at $t=0$ and then starts expanding with the initial velocity $R_{1}$. The cylinder penetrates the liquid in the interval $0<t<t_{1}$, where $R^{\prime}\left(t_{1}\right)=0$. In this problem, the time $t_{1}$, initial radius $R_{0}$ and $k=R\left(t_{1}\right) / R_{0}$ are given (see [8] for more details of the problem formulation). Starting from $t=t_{1}$ the cylinder is contracting, which corresponds to the exit of the cylinder from the liquid (see Figure 6). 


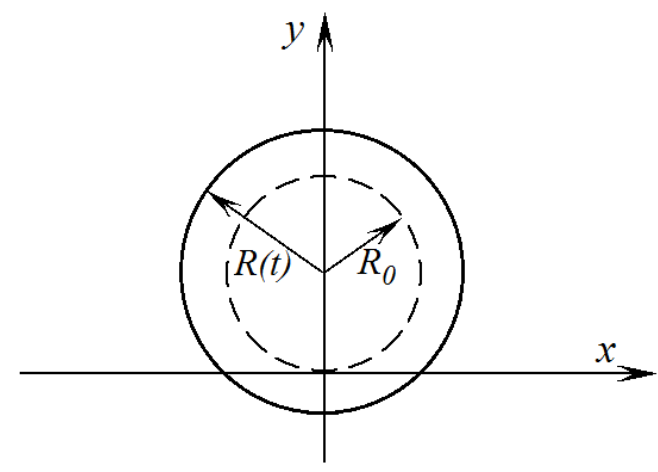

Fig. 6 Sketch of expanding and contracting circular cylinder.

The non-dimensional hydrodynamic force acting on the cylinder,

$$
F^{*}\left(t^{*}\right)=F\left(t^{*} t_{1}\right) \frac{t_{1}^{2}}{4 \rho R_{0}^{3}(k-1)^{2}},
$$

is evaluated as a function of the non-dimensional time $t^{*}=t / t_{1}$ by using equations (14) and (15) of the Wagner model during the expansion stage and the equations (53)-(55) during the contraction stage.

Equation (14) for the size of the contact region during the expansion stage, $0<t<t_{1}$, and the shape function (62) provide

$$
\mathbf{E}\left(\frac{c(t)}{R(t)}\right)=\frac{\pi}{2} \frac{R_{0}}{R(t)},
$$

where $\mathbf{E}(m)=\int_{0}^{\pi / 2} \sqrt{1-m^{2} \sin ^{2} \theta} d \theta$ is the complete elliptic integral of the second kind. In the non-dimensional variables, $R(t)=R_{0} r\left(t^{*}\right), r\left(t^{*}\right)=1+(k-1)\left(2 t^{*}-t^{* 2}\right), c(t)=m R(t)$, where $m\left(t^{*}\right)$ is the solution of the equation $\mathbf{E}(m)=\pi /\left(2 r\left(t^{*}\right)\right)$. The force evaluated for the shape functions (62) is given by

$$
\begin{gathered}
F^{*}\left(t^{*}\right)=2 r\left(t^{*}\right)\left(1-t^{*}\right)^{2}\left(\frac{m^{2} \mathbf{K}^{2}(m)}{\mathbf{K}(m)-\mathbf{E}(m)}+\left(m^{2}-2\right) \mathbf{K}(m)+2 \mathbf{E}(m)\right) \\
-\frac{r^{2}\left(t^{*}\right)}{k-1}\left(\left(m^{2}-1\right) \mathbf{K}(m)+\mathbf{E}(m)\right)
\end{gathered}
$$

and for the parabolic approximation with $y_{b}(x, t) \approx x^{2} /(2 R(t))+R_{0}-R(t)$ by using $(22)$ with $h(t)=R_{0}-R(t)$,

$$
F_{p a r}^{*}\left(t^{*}\right)=\pi\left[\left(1-t^{*}\right)^{2}\left(6 r\left(t^{*}\right)-4\right)-\left(3 r\left(t^{*}\right)-1\right)\left(t^{*}-t^{* 2} / 2\right)\right],
$$

where $\mathbf{K}(m)$ is the complete elliptic integral of the first kind and $0<t^{*}<1$. During the contraction stage, $t_{1}<t<t_{2}$, the hydrodynamic force is given by (53) which provides

$$
\begin{aligned}
F^{*}\left(t^{*}\right)= & 2 r\left(t^{*}\right)\left(t^{*}-1\right)^{2}\left(\left(m^{2}-2\right) \mathbf{K}^{2}(m)+2 \mathbf{E}(m)\right)- \\
& -\frac{r^{2}\left(t^{*}\right)}{k-1}\left(\left(m^{2}-1\right) \mathbf{K}(m)+\mathbf{E}(m)\right),
\end{aligned}
$$


where $m=c(t) / R(t)$ and $c(t)$ is computed by (54) and (55) for the shape function (62).

In the parabolic approximation, the force is given by

$$
F_{p a r}^{*}\left(t^{*}\right)=-\frac{\pi}{32} m^{2} r\left(t^{*}\right)\left[4 m^{2}\left(t^{*}-1\right)^{2}+\frac{8-m^{2}}{k-1} r\left(t^{*}\right)\right] .
$$

where $m=c(t) / R(t)$. The size of the contact region, $c(t)$, is provided by (54) and (55), where now

$$
\begin{gathered}
y_{b, t t}(x, t)=\frac{x^{2}}{2}\left(\frac{1}{R}\right)^{\prime \prime}-R^{\prime \prime}(t), \\
H(\sigma, t(\sigma))=\frac{c_{0}^{2}(1-\sigma)}{4}\left(\frac{1}{R}\right)^{\prime \prime}-R^{\prime \prime}(t), \quad L(\sigma, \alpha, t(\alpha))=\frac{\pi c_{0}^{2}}{4}\left(\frac{1}{R}\right)^{\prime \prime}(t(\alpha)) .
\end{gathered}
$$

Equations (65) and (67) at $t^{*}=1$ give $F_{\text {par }}^{*}(1)=-\pi(3 k-1) / 2$. Asymptotic analysis of equations (64)-(67) for $k \rightarrow 1$, when the linear model and the parabolic approximations are justified, provides

$$
F^{*}(1)-F_{p a r}^{*}(1) \sim \frac{3}{4} \pi(k-1) .
$$

Therefore, the accuracy of the parabolic approximation is of order $O(k-1)$ as $k \rightarrow 1$. The hydrodynamic forces (64)-(67) obtained within the present linearised models of water entry and exit are compared with the CFD and theoretical predictions from [3] in Figure 7 for $R=0.65 \mathrm{~m}, t_{1}=0.5 \mathrm{sec}, k=1.025$ and $k=1.1$. For $k=1.1$ the cylinder expands from $R_{0}=0.65 \mathrm{~m}$ to $R\left(t_{1}\right)=0.715 \mathrm{~m}$ in $0.5 \mathrm{~s}$. Equation (19) yields that $c\left(t_{1}\right) \approx 0.43 \mathrm{~m}$ at the end of the expansion stage. This result implies that the size of the contact region for $k=1.1$ is comparable with the radius of the cylinder and that the nonlinear effects matter. For $k=1.025$ we have $c\left(t_{1}\right) \approx 0.2 \mathrm{~m}$ and the present linearised model is applicable.

Figure 7 shows that the linearised model of water exit predicts the forces which are closer to the CFD results than the predictions by the von Karman model from [8]. The theoretical force in [8] was computed by using the Modified Logvinovich Model (MLM) [9] for $0<t^{*}<1$ and the von Karman approach [18] for the contraction stage. Here $t^{*}=1$ correspond to the beginning of the contraction stage. The parabolic approximation of the circular cylinder provides the forces which are very close to those calculated for the actual shape function (62). However, the minimum of the force at $t^{*}=1$ is overpredicted by the linearised model. Note that the boundary conditions in the linearised model are imposed on the equilibrium level of the liquid and are linearised at this level.
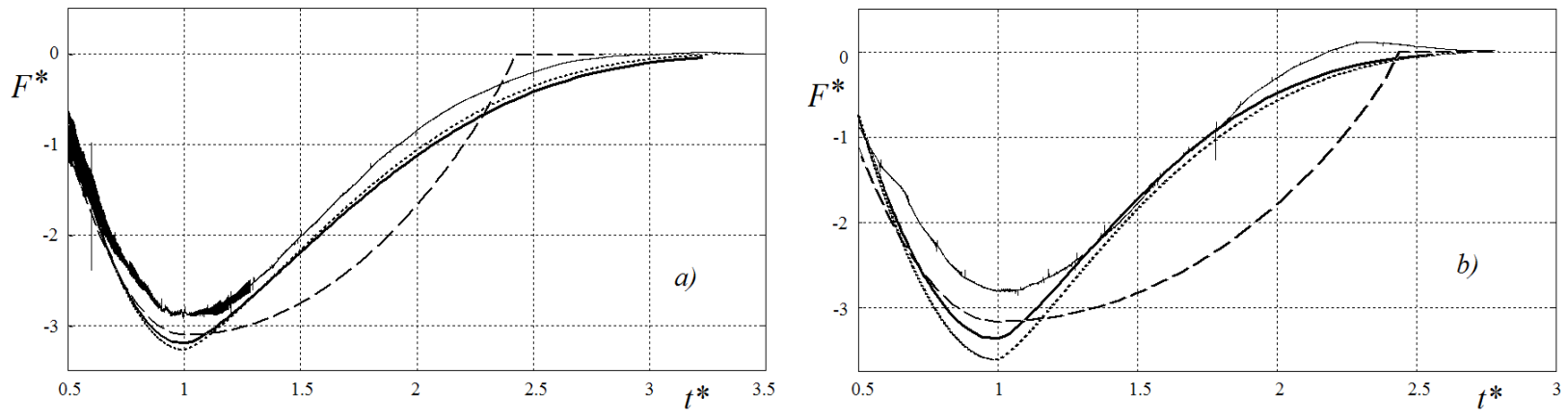

Fig. 7 The non-dimensional hydrodynamic force acting on the expanding / contracting cylinder as a function of the non-dimensional time $t^{*}$ for $(a) k=0.025$ and $(b) k=1.1$. The CFD results from [8] are shown by thin black lines, the theoretical predictions from [8] are shown by large dashed lines. The force by the present model (64), (66) is shown by thick black lines, and the corresponding parabolic approximation (65), (67) is shown by the dotted lines. 


\section{Weakly nonlinear model of water exit}

In this section, we estimate the hydrodynamic loads during water exit by taking into account some non-linear effects and the shape of the body. The ideas from the Modified Logvinovich Model [9] and Generalized Wagner Model [19] of water entry are used.

\subsection{Pressure and Force on Body}

Combining the Bernoulli equation (2) and the original condition, $\varphi_{y}=y_{b, x} \varphi_{x}+y_{b, t}$, on the wetted part of the moving body surface Eq.(1), we obtain the pressure distribution along the wetted surface

$$
p\left(x, y_{b}(x, t), t\right)=-\rho\left(\phi_{t}-\frac{y_{b, t} y_{b, x}}{1+y_{b, x}^{2}} \phi_{x}+\frac{1}{2} \frac{\phi_{x}^{2}-y_{b, t}^{2}}{1+y_{b, x}^{2}}\right),
$$

where $\phi(x, t)=\varphi\left(x, y_{b}(x, t), t\right)$ is the velocity potential on the wetted surface (see [9] for more details). Within the linearized model of water exit the right-hand side in Eq.(68) is approximated by $-\rho \phi_{t}(x, t)$ and $\phi_{t}(x, t) \approx \varphi_{t}(x, 0, t)$, where $\varphi_{t}$ is the solution of the boundary-value problem (3)-(6). Within the MLM [9], we keep all terms in Eq.(68) and approximate $\phi(x, t)$ by the two-terms Taylor expansion, $\phi(x, t) \approx \varphi(x, 0, t)+$ $\varphi_{y}(x, 0, t) y_{b}(x, t)$, where $\varphi_{y}(x, 0, t) \approx y_{b, t}(x, t)$ and $\varphi(x, y, t)$ is the solution of Eqs. (3)-(6).

The new weakly nonlinear model of water exit is based on Eq.(68), where the terms with $\phi_{x}$ and $y_{b, x}^{2}$ are neglected similar to the linearized exit model [5] and the derivative $\phi_{t}(x, t)$ is approximated by the two-terms Taylor expansion about the splash-up level $y=y_{b}(c(t), t)$,

$$
\phi_{t}(x, t) \approx \varphi_{t}(x, 0, t)+\varphi_{t y}(x, 0, t)\left(y_{b}(x, t)-y_{b}(c, t)\right),
$$

similar to the model from [19]. Here $\varphi_{t}(x, 0, t)$ is the solution of Eqs.(3)-(6) and $\varphi_{t y}(x, 0, t)=$ $y_{b, t t}(x, t)$. Note that the boundary conditions are linearized and imposed now on the splash-up height $y_{b}(c, t)$. The linear problem (3)-(6) is not affected by the height at which the boundary conditions are imposed. Therefore, the potential $\varphi$ in Eq.(69) does not depend on the level, at which the linearization is performed, and this level enters Eq.(69) only through the term $y_{b}(c, t)$. The formula for the pressure distribution reads now

$$
p\left(x, y_{b}(x, t), t\right)=-\rho \varphi_{t}(x, 0, t)-\rho\left(y_{b, t t}(x, t)\left(y_{b}(x, t)-y_{b}(c, t)\right)-\frac{1}{2} y_{b, t}^{2}(x, t)\right) .
$$

The first term in Eq. $(70),-\rho \varphi_{t}(x, 0, t)$, corresponds to the pressure distribution provided by the linearized exit model. The hydrodynamic force is decomposed as

$$
F(t)=F_{L}(t)+F_{b}(t),
$$

where $F_{L}(t)$ is the force predicted by the linearized exit model and $F_{b}(t)$ is the correction term,

$$
F_{b}(t)=\rho \int_{0}^{c(t)}\left(y_{b, t}^{2}(x, t)-2 y_{b, t t}\left(y_{b}(x, t)-y_{b}(c, t)\right)\right) d x,
$$

and the size of the contact region $c(t)$ is given by Eqs. (54), (55). The present model of the hydrodynamic force (71), (72) is simpler than that by MLM [9] but more accurate compared with the linearized model from [5]. 


\subsection{Results for Expanding and Contracting Cylinder}
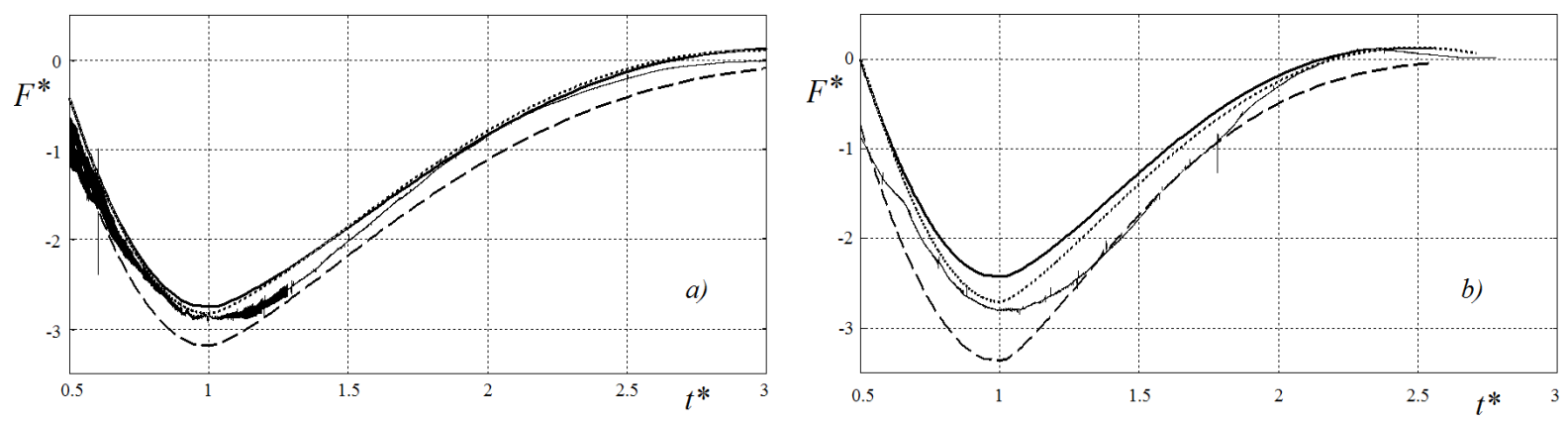

Fig. 8 The non-dimensional hydrodynamic force acting on the expanding/contracting cylinder as a function of the non-dimensional time $t^{*}$ for (a) $k=1.025$ and (b) $k=1.05$. The CFD results from [8] are shown by thin black lines, the theoretical predictions $F_{L}^{*}$ are shown by the dashed lines. The force by the nonlinear model Eqs.(71), (72) is shown by thick black

lines, and the corresponding non-linear parabolic approximation is shown by dotted lines.

The formulae (71), (72) are applied to the problem of expanding/contracting cylinder described in Section 6 during both the expansion and contraction stages. The forces predicted by the weakly nonlinear model $(71),(72)$ are compared with both the forces $F_{L}(t)$ by the linearized model and the forced by CFD in Fig. 8 for $k=1.025$ and $k=1.05$. It is seen that the correction term $F_{b}(t)$ improves the comparison of the theoretical predictions with the numerical results by CFD. The theoretical results are obtained by using the parabolic approximation. Calculations with the actual shape of the cylinder (62) were also performed to demonstrate that the accuracy of the parabolic approximation is of order $O(k-1)$ as $k \rightarrow 1$.

\section{Forces on a planing ellipsoid}

The developed models are applied to the three-dimensional steady problem of a rigid ellipsoid (see Fig. 1)

$$
x^{2} / b^{2}+\left(y-h_{0}\right)^{2} / c^{2}+(z-V t)^{2} / a^{2}=1 .
$$

The ellipsoid (73) with semi-axes $a, b$ and $c$ is slightly submerged at $c-h_{0}$ and moves along the water surface with constant speed $V$ in the $z$-direction. The problem is considered within the 2D+T approximation (see Section 2). The hydrodynamic loads are determined for each section of the body by using the Wagner theory, if the section penetrates water, and the linearized exit model, if the section exits from the water. The correction (72) is applied. The control plane is introduced at $z=a$. The forces are calculated for the two-dimensional sections

$$
y=y_{b}(x, t)=h_{0}-c \sqrt{\tau(2-\tau)-x^{2} / b^{2}}, \quad \tau=V t / a,
$$

which are approximated by the parabolic shapes $y_{b}(x, t) \approx x^{2} /(2 R(t))+h(t)$. The distributions of both the pressure at $x=0$ and sectional forces are shown in Fig. 9 together with CFD results obtained within the Navier-Stokes three-dimensional model without gravity and surface tension. Calculations were performed for $a=10 \mathrm{~m}, b=c=1 \mathrm{~m}$ and 
$V=50 \mathrm{~m} / \mathrm{s}$. The present linearized model with the correction (72) over-predicts the loads for the sections in entry but well corresponds to the CFD predictions for the sections in exit. The loads for the sections in entry can be potentially improved by using the MLM and a local three-dimensional model close to the jet overturning region. This improvement of the loads for the entry stage has not been pursued here. The present analysis is focused on the models of the exit stage.
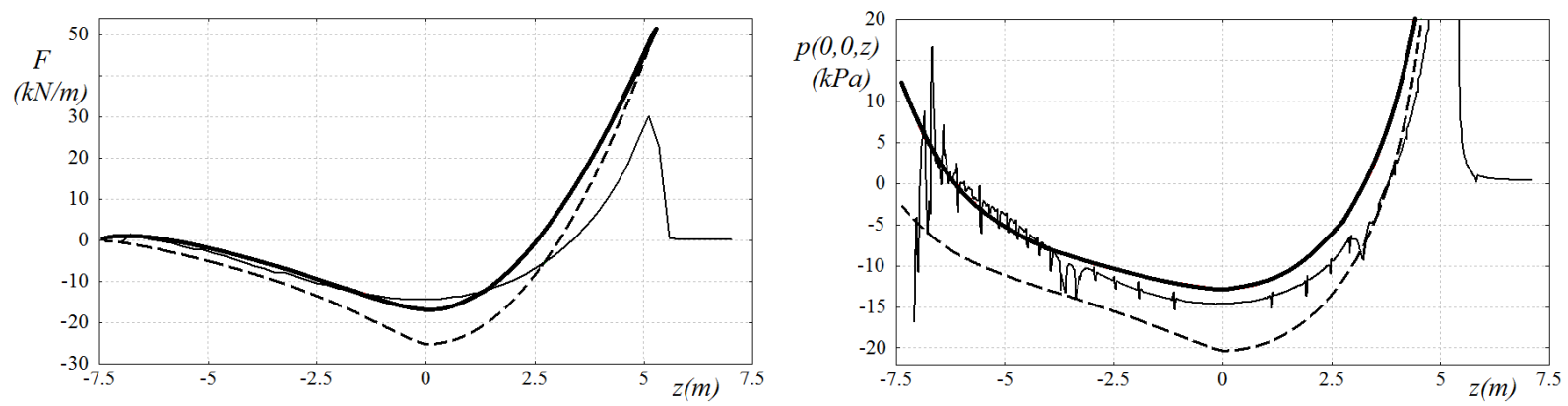

Fig. 9 The hydrodynamic sectional force (a) and the pressure along the keel, $x=0$, (b) as functions of the longitudinal coordinate $z$. The CFD results are shown by the thin black line, the theoretical predictions for the parabolic approximation by the linearized entry/exit model are shown by dashed lines and by the weakly nonlinear model are shown by thick black lines.

The total vertical force $F_{v}$ acting on the moving ellipsoid is evaluated by Eqs.(8)(10) both for the linear model and weakly non-linear model of Section 7 . The force is decomposed into the force acting on the sections entering water, from the nose of the body to its middle, $F_{v i}$, and the force, $F_{v e}$, acting on the sections which exit from the water within the $2 \mathrm{D}+\mathrm{T}$ approach. The force components, $F_{v i}$ and $F_{v e}$, are computed within the linearized entry/exit model and the weakly nonlinear model. Within the linearized Wagner model of water entry $F_{v i}=0$, as it was shown in Section 2. The numerical values within the linearized model are $F_{v i} \approx 0.2 \mathrm{kN}$ and $F_{v e} \approx-77.05 \mathrm{kN}$ giving the total force $F_{v} \approx-76.85 \mathrm{kN}$. The corresponding values for the weakly nonlinear model are $F_{v i} \approx 34.4 \mathrm{kN}, F_{v e} \approx-46.7 \mathrm{kN}$ and $F_{v} \approx-12.3 \mathrm{kN}$. The total force predicted by CFD calculations is larger in magnitude then $-12.3 \mathrm{kN}$. The hydrodynamic torque $M$ acting on the moving ellipsoid is also decomposed in two components, $M=M_{i}+M_{e}$, according to equation (12). The linearized entry/exit model predicts $M_{i} \approx 175.4 \mathrm{kN} \mathrm{m}$, $M_{e} \approx 165.6 \mathrm{kNm}$ and $M \approx 341 \mathrm{kNm}$. The weakly nonlinear model provides $M_{i} \approx$ $253 \mathrm{kNm}, M_{e} \approx 80.33 \mathrm{kNm}$ and $M \approx 333.33 \mathrm{kNm}$. Despite the components $M_{i}$ and $M_{e}$ are rather different in these two models, the total torque, $M$, is well predicted by the linearized model. The negative hydrodynamics loads acting in rear part of the body and described by the exit models significantly contribute to the total torque.

The shape of the contact region between the ellipsoid (73) and water is given by the Wagner model of water entry, where $z-V t>0$, and by the linearized model of water exit (see Section 6), where $z-V t<0$. The boundary of this region is shown in Fig.10 by the red line. As a reference, the intersection line between the ellipsoid and the equilibrium water level, $y=0$, is shown in blue. The Fig. 10 shows that the actual wetted area of this planing body is bigger than that expected without account for the elevation of the free surface. 


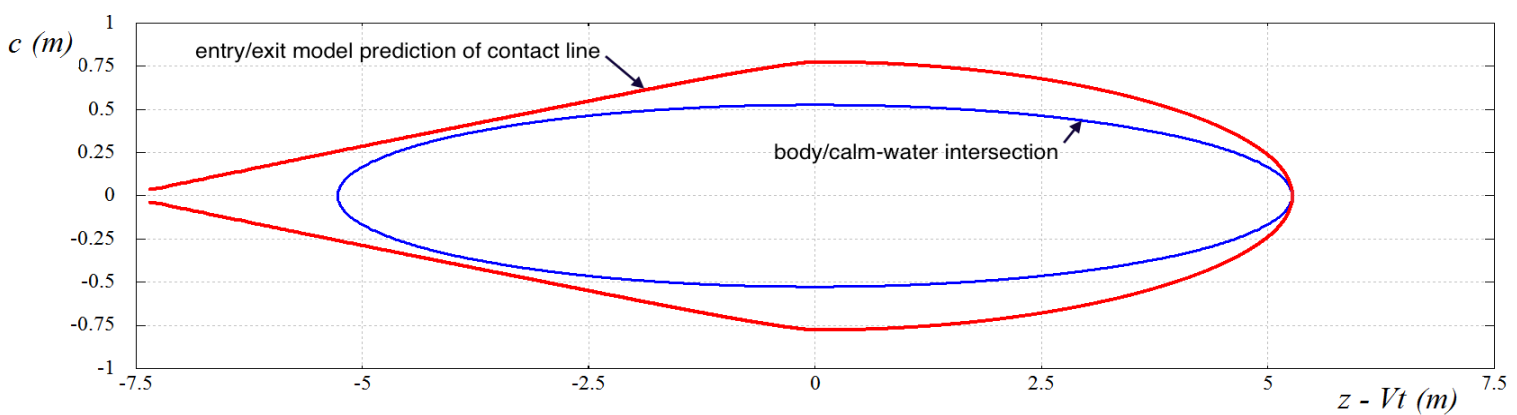

Fig. 10 The wetted part of the body surface predicted by the linearized entry/exit model (red line) and without account for the free surface deformations (blue line) in the moving coordinate system. 


\section{Conclusion}

The linearized model of water exit [5] has been generalized to include body motions with time-dependent acceleration and lifting of a body with time-varying shape from a liquid surface. A weakly non-linear model of water exit has been presented. The later model accounts for some nonlinear terms in the Bernoulli equation for the hydrodynamic pressure and the shape of the body. The model employs the solution of the linearized exit problem and the size of the wetted area of the body surface predicted by the linearized model of water exit. It was shown that the weakly nonlinear model of water exit predicts the hydrodynamic loads which are closer to the numerical loads by CFD than the predictions by the linearized model of water exit. The developed models of water exit were combined with the $2 \mathrm{D}+\mathrm{T}$ approximation and applied to the steady problem of an elongated ellipsoid moving along the water free surface. The obtained theoretical results were compared with the three-dimensional numerical simulations by a Navier-Stokes solver from Open FOAM library in terms of sectional forces and the pressure distribution along the keel of the moving body. The hydrodynamic loads on the body sections behind the middle of the ellipsoid are well predicted by the present weakly nonlinear model of water exit. The theoretical loads acting on the front part of the ellipsoid from the nose to the middle of it are rather different from the CFD results. The loads on this part of the body were evaluated by using the original linearized Wagner model of water entry. More accurate models, such as MLM and the Generalized Wagner Model, can significantly improve the theoretical predictions.

Three-dimensional unsteady problems of a smooth elongated body moving along the water surface can be solved by using the exit models of the present study. The unsteady problems are of interest for analysis of aircraft ditching (emergency landing on water) and performance of high-speed boats. Unsteady motions of such bodies in uni-directional waves (head and following sea) can be also described by the entry/exit models of this paper.

The entry and exit models of the present paper cannot be used for large penetrations of the planing body. The forces on the front sections of the body, which are in entry, can be calculated by using the Generalized Wagner Model [19] with account for the flow separation from the surfaces of the sections. The sections in the rear part of the body, which are in exit, require more elaborated models if the section exits water from large depth.

Acknowledgement: This work has been supported by the NICOP research grant "Fundamental Analysis of the Water Exit Problem" N62909-13-1-N274, through Dr. Woei-Min Lin. This work has also been supported by the Office of Naval Research Young Investigator Award N00014-14-1-0577, monitored by Kelly Cooper. Any opinions, findings, and conclusions or recommendations expressed in this material are those of the authors and do not necessarily reflect the views of the Office of Naval Research. Preliminary results of this work were reported at the International Workshops on Water Waves and Floating Bodies in Osaka 2014 [14] and in Bristol 2015 [20]. 


\section{References}

[1] Camilleri, J., Temarel, P., and Taunton, D. Two-dimensional numerical modelling of slamming impact loads on high-speed craft. In 7th International Conference on Hydroelasticity in Marine Technology, Split, Croatia, September 16-19 2015.

[2] Facci, A. L., Panciroli, R., Ubertini, S., and Porfiri, M. Assessment of PIV-based analysis of water entry problems through synthetic numerical datasets. Journal of Fluids and Structures, 55:484-500, 2015.

[3] Facci, A. L., Porfiri M., and Ubertini, S.. Three-dimensional water entry of a solid body: A computational study. Journal of Fluids and Structures, 66:36-53, 2016.

[4] Southall, N., Choi, S., Lee, Y., Hong, C., Hirdaris, S., and White, N. Impact analysis using CFD - a comparative study. In Proceedings of the Twenty-fifth (2015) International Ocean and Polar Engineering Conference, Kona, Big Island, Hawaii, USA, June 21-16 2015.

[5] Korobkin, A. A. (2013). A linearized model of water exit. Journal of Fluid Mechanics, $737,368-386$.

[6] Fontaine, E., Cointe, R. (1997). A slender body approach to nonlinear bow waves. Philosophical Transactions of the Royal Society of London. Series A: Mathematical, Physical and Engineering Sciences, 355(1724), 565-574.

[7] Fontaine, E., Faltinsen, O.M., Cointe, R. (2000). New insight into the generation of ship bow waves. Journal of Fluid Mechanics, 421, 15-38.

[8] Tassin, A., Piro, D. J., Korobkin, A. A., Maki, K. J., Cooker, M. J. (2013). Twodimensional water entry and exit of a body whose shape varies in time. Journal of Fluids and Structures, 40, 317-336.

[9] Korobkin, A. (2004). Analytical models of water impact. Eur. Journal of Applied Mathematics, 15(06), 821-838.

[10] Piro, D. J., Maki, K. J. (2011). Hydroelastic wedge entry and exit. In Eleventh International Conference on Fast Sea Transport. 26-29 September, Honolulu, Hawaii, USA.

[11] Piro, D. J., Maki, K. J. (2012). Water exit of a wedge-shaped body. In 27th IWWWFB, Copenhagen, Denmark.

[12] Piro, D. J., Maki, K. J. (2013). Hydroelastic analysis of bodies that enter and exit water. Journal of Fluids and Structures, 37, 134-150.

[13] D. J. Piro and K. J. Maki. An adaptive interface compression method for water entry and exit. Technical Report 350, University of Michigan Department of Naval Architecture and Marine Engineering, March 2013.

[14] Korobkin A.A., Khabakhpasheva T.I., Maki K.J. (2014). Water-exit problem with prescribed motion of a symmetric body // Proc. 29-th Intern. Workshop on Water Waves and Floating Bodies (Ed.: M. Kashiwagi, M.Minoura) March 30-April 2, 2014, Suita, Osaka, Japan, p. 117-120 
[15] Rajavaheinthan, R., Greenhow, M. (2015). Constant acceleration exit of twodimensional free-surface-piercing bodies. Applied Ocean Research, 50, 30-46.

[16] Wagner H. (1932) Über Stoss- und Gleitvergänge an der Oberfläche von Flüssigkeiten // Z. Angew. Mach. Mech. Vol.12. H.4. S.193-235.

[17] Korobkin A.A. (1996) Water impact problems in ship hydrodynamics. In: Advances in Marine Hydrodynamics (ed. M. Ohkusu). Southampton: Computational Mechanics Publications, p.323-371.

[18] Von Kármán T. (1929) The impact of seaplane floats during landing // NACA, Technical Note 321, Washington, DC, USA. 1929. p. 309-313.

[19] Zhao R, Faltinsen O, Aarsnes J. (1996) Water entry of arbitrary two-dimensional sections with and without flow separation. Proc. 21th symposium on naval hydrodynamics, Trondheim, Norway.

[20] Khabakhpasheva T.I.,Korobkin A.A., Maki K.J. (2015). A linearized exit model for prediction of forces on a body within the 2D+T framework // Proc. 30-th Intern. Workshop on Water Waves and Floating Bodies (Eds: R.Porter, Jun Zang) April 12-15, 2015, Bristol, UK, p.105-108.

[21] E. Berberović, N. P. van Hinsberg, S. J. I. V. Roisman, and C. Tropea. Drop impact onto a liquid layer of finite thickness: Dynamics of the cavity evolution. Physical Review E, 79, 2009. 